\title{
General Formula for the Magnetoresistance on the Basis of the Fermi Liquid Theory
}

\author{
Hiroshi KOnTANI ${ }^{1,2}$ \\ ${ }^{1}$ Theoretische Physik III, Elektronische Korrelationen und Magnetismus, Universität Augsburg, D-86135 Augsburg, Germany. \\ 2 Department of Physics, Saitama University, 255 Shimo-Okubo, Urawa-city, 338-8570, Japan.
}

(November 5, 2018)

\begin{abstract}
The general expression for the magnetoresistance (MR) due to the Lorentz force is derived by using the Fermi liquid transport theory based on the Kubo formula. The obtained gauge-invariant expression is exact for any strength of the interaction, as for the most singular term with respect to $1 / \gamma_{k}^{*}\left(\gamma_{k}^{*}\right.$ being the quasiparticle damping rate). By virtue of the exactness, the conserving laws are satisfied rigorously in the present expression, which is indispensable for avoiding unphysical solutions. Based on the derived expression, we can calculate the MR within the framework of the Baym-Kadanoff type conserving approximation, by including all the vertex corrections required by the Ward identity. The present expression is significant especially for strongly correlated systems because the current vertex corrections will be much important. On the other hand, if we drop all the vertex corrections in the formula, we get the MR of the relaxation time approximation (RTA), which is commonly used because of the simplicity. However, the RTA is dangerous because it may give unphysical results owing to the lack of conserving laws. In conclusion, the present work enables us to study the MR with satisfying the conserving laws which is highly demanded in strongly correlated electrons, such as high- $T_{\mathrm{c}}$ superconductors, organic metals, and heavy Fermion systems. In Appendix D, we reply to the comment by O. Narikiyo cond-mat/0006028. (Note that Appendix $\mathrm{D}$ exists only in the e-preprint version.)
\end{abstract}

PACS numbers: 72.10.Bg, 74.25.Fy, 74.70.-h

\section{INTRODUCTION}

The transport phenomena under the magnetic field are very important and interesting field in condensed matter physics. Especially, the magnetoresistance (MR) is of current interest: It has been attracting great attentions of many researchers because it gives us rich information on the electronic structure of the system. There are several possible mechanisms of the MR, for example, (i) the Lorentz force on conduction electrons (orbital effect), (ii) the doubleexchange mechanism (spin effect), and (iii) the Anderson localization mechanism in dirty metals, or the Kondo effect caused by the magnetic impurities. It is well-known that the huge negative MR due to (ii) is realized in Mn-oxides at the ferromagnetic transition temperature, i.e., the colossal magnetoresistance. The negative MR due to (iii) is observed in dirty semiconductors.

On the other hand, in usual pure paramagnetic metals, the positive MR due to (i) is the most dominant, which is called the orbital MR. There is a long-history on the study of the orbital MR based on the Boltzmann transport theory [1]. In many theoretical studies, the collision term in the Boltzmann equation is simply approximated by introducing the phenomenological relaxation time $\left(\tau_{\mathbf{k}}\right)$, which is called the relaxation time approximation (RTA). There, the correlation effect between particle-hole pair is totally dropped. Although RTA is a quasiclassical approximation, various important and useful results have been obtained. For example, the MR as a function of the angle between $\vec{B}$ and $\vec{I}$ in various metals was reproduced within the RTA [1].

Especially, the Kohler's rule is one of the most essential relations derived by the RTA: According to this rule, the conductivity $\sigma_{\mu \nu}$ in the magnetic field $\vec{B}$ is represented in the functional form $\sigma_{\mu \nu}=\tau \cdot F_{\mu \nu}(\tau B)$, if the anisotropy of $\tau_{\mathbf{k}}$ is not so large. According to the Onsager's reciprocity theorem, $F_{\mu \nu}(x)$ is an even-function of $x$ as for the diagonal conductivity $(\mu=\nu)$, and it is an odd-function as for the off-diagonal conductivity $(\mu=x, \nu=y)$.

By definition, the resistance without the magnetic filed $\left(\rho_{0}\right)$, the Hall coefficient $\left(R_{\mathrm{H}}\right)$, and the magnetoresistance $\left(\Delta \rho / \rho_{0}\right)$, are given by

$$
\begin{aligned}
\rho_{0} & =1 / \sigma_{0} \\
R_{\mathrm{H}} & =\left(\Delta \sigma_{x y} / B\right) / \sigma_{0}^{2}, \\
\Delta \rho / \rho_{0} & =-\Delta \sigma_{x x} / \sigma_{0}-\left(\Delta \sigma_{x y} / \sigma_{0}\right)^{2},
\end{aligned}
$$


where $\sigma_{0}$ is the diagonal conductivity without $\vec{B} . \Delta \sigma_{x y}$ and $\Delta \sigma_{x x}$ are components proportional to $B$ and $B^{2}$, respectively. We call $\Delta \sigma_{x x}$ the magnetoconductivity (MC). As a result, the Kohler's rule predicts the relations $\rho_{0} \propto \tau, R_{\mathrm{H}} \propto \tau^{0}$, and $\Delta \rho / \rho_{0} \propto \tau^{2} \cdot B^{2}$. These relations are well satisfied in various ordinary metals, which may be interpreted as nearly free electron systems.

Interestingly, however, the Kohler's rule is strongly violated in several strongly correlated metals according to recent experimental studies. For example, $R_{\mathrm{H}}$ in the normal state in high- $T_{\mathrm{c}}$ cuprates is approximately proportional to $T^{-1}$, and $\Delta \rho / \rho_{0} \propto \rho_{0}^{-2} \cdot T^{-2}$. These seemingly non-Fermi liquid behaviors of the transport phenomena in high- $T_{\mathrm{c}}$ cuprates have been studied as a central issue both theoretically and experimentally because they should reflect the fundamental electronic property of high- $T_{\mathrm{c}}$ cuprates.

Up to now, several authors have studied the transport phenomena in high- $T_{\mathrm{c}}$ cuprates based on the RTA, and claimed that the strong anisotropy of $\tau_{k}$ can explain the observed violation of the Kohler's rule. [2]. However, its effect is too insufficient to reproduce the prominent non-Fermi liquid behaviors observed for wider range of temperatures. On the other hand, we recently studied the $R_{\mathrm{H}}$ in high- $T_{\mathrm{c}}$ cuprates based on the general formula for $\Delta \sigma_{x y}$ derived by Ref. [3], which is formally exact of order $\tau^{2}$. Then, we find out that the vertex corrections for the current, which are dropped in the RTA, cause the Curie-Weiss like behavior of $R_{\mathrm{H}}$ in the presence of AF fluctuations [4].

The aim of this paper is to derive the general expression for the $M C$ from the Kubo formula. The obtained expression for $\Delta \sigma_{x x}$, which is formally exact in order $\tau^{3}$, contains all the vertex corrections which ensure the conserving laws. In the system with strong correlations, the role of the vertex corrections will qualitatively change the behavior of the $\mathrm{MR}$, as it does for the Hall effect. In this respect, the RTA is unreliable because all the current vertex corrections are neglected there. Based on the derived expression, we will study the MR in high- $T_{\mathrm{c}}$ cuprates and discuss the violation of Kohler's rule in later publications [8].

In the absence of the magnetic field, Eliashberg derived in 1961 the expression for the conductivity of the Fermi liquid system from the Kubo formula [9]:

$$
\sigma_{x x}=\frac{e^{2}}{v_{B}} \int_{\mathrm{FS}} \frac{d S_{k}}{\left|\vec{v}_{\mathbf{k}}\right|} v_{\mathbf{k} x} J_{\mathbf{k} x} \cdot 2 \tau_{\mathbf{k}}
$$

which is exact up to $O(\tau)$. $\int_{\mathrm{FS}} d S_{k}$ means the two-dimensional integration on the Fermi surfaces, and $v_{B}=(2 \pi)^{3}$ in the cubic lattice. $\tau_{\mathbf{k}} \equiv 1 /\left(-2 \operatorname{Im} \Sigma_{\mathbf{k}}(+\mathrm{i} 0)\right)>0$, where $\Sigma_{\mathbf{k}}(\omega)$ is the self-energy. $v_{\mathbf{k} x} \equiv\left(\partial / \partial k_{x}\right)\left(\epsilon_{\mathbf{k}}^{0}+\operatorname{Re} \Sigma_{\mathbf{k}}(0)\right)$ is the quasiparticle velocity without the renormalization factor $z_{k}$. Moreover, $J_{\mathbf{k} x}$ has the four-point vertex correction $\mathcal{T}_{22}$, which is not given by the self-energy through the Ward identity. ( $J_{\mathbf{k} x}$ is given by eq.(70) in this paper.) This non-trivial current vertex correction from $\mathcal{T}_{22}$ is indispensable to avoid unphysical results. For instance, Yamada and Yosida proved that $\sigma_{x x}$ given by eq.(2) diverges unless any Umklapp scattering processes exist, reflecting the momentum conservation law [10]. This physically reasonable result cannot be reproduced if we drop the vertex correction from $\mathcal{T}_{22}$.

The exact expression for the Hall conductivity of order $\tau^{2}$ was given by Kohno-Yamada as follows: [3]

$$
\Delta \sigma_{x y}=-B \cdot \frac{e^{3}}{2 v_{B}} \int_{\mathrm{FS}} \frac{d S_{k}}{\left|\vec{v}_{\mathbf{k}}\right|} v_{\mathbf{k} x}\left[J_{\mathbf{k} x} \frac{\partial J_{\mathbf{k} y}}{\partial k_{y}}-J_{\mathbf{k} y} \frac{\partial J_{\mathbf{k} x}}{\partial k_{y}}\right]\left(2 \tau_{\mathbf{k}}\right)^{2},
$$

where the uniform magnetic field is along the $z$-axis. Here the carrier of an electron is $-e(e>0)$. Note that if we replace $J_{\mathbf{k} \mu}$ with $v_{\mathbf{k} \mu}$ in eqs. (2) and (3), we get the results of the RTA.

As for $\Delta \sigma_{x y}$, the vertex corrections in $J_{\mathbf{k} x}$ also play important roles in strongly correlated systems. In fact, according to recent theoretical studies, the vertex corrections in $J_{\mathbf{k} x}$ give rise to the strong enhancement of $R_{\mathrm{H}}$ in nearly AF Fermi liquids, like in high- $T_{\mathrm{c}}$ superconductors or in $\kappa$-(BEDT-TTF) organic superconductors [1:7]. Noteworthily, $R_{\mathrm{H}}$ becomes negative in electron-doped compounds irrespective of the fact that its Fermi surface is hole-like everywhere [11]. This long-standing mystery, which cannot be explained within the RTA, is naturally reproduced in Refs. [4] and 50] due to the fact that $\vec{J}_{\mathbf{k}}$ is no more parallel to $\vec{v}_{\mathbf{k}}$.

The purpose of this article is to derive the conserving expression for the magnetoconductivity $\Delta \sigma_{x x}$ which is exact in order $\tau^{3}$. We notice that the magnetoconductivity within the RTA, $\Delta \sigma_{x x}^{\mathrm{RTA}}$, is given by

$$
\Delta \sigma_{x x}^{\mathrm{RTA}}=-B^{2} \cdot \frac{e^{4}}{4 v_{B}} \int_{\mathrm{FS}} \frac{d S_{k}}{\left|\vec{v}_{\mathbf{k}}\right|}\left\{\left(\vec{v}_{\mathbf{k}} \times \hat{e}_{z}\right) \cdot \vec{\nabla}\left(2 \tau_{\mathbf{k}} v_{\mathbf{k} x}\right)\right\}^{2}\left(2 \tau_{\mathbf{k}}\right),
$$

where $\hat{e}_{z}$ is the unit vector along the magnetic field [1]. Note that the expression (4) has the contribution from the $k$-derivative of $\tau_{\mathbf{k}}$, while $\sigma_{x x}$ and $\Delta \sigma_{x y}$ given by eqs. (2) and (3) do not have it. $\Delta \sigma_{x x}^{\text {RTA }}$ given by eq. (4) will be unreliable in correlated electron systems because conserving laws are violated in the sense of Baym and Kadanoff [12]. Whereas, the derived formula for $\Delta \sigma_{x x}$ in this paper, eq.(77) or eq.(78) in $\S \mathrm{V}$, is conserving and exact up to $O\left(\tau^{3}\right)$. 
In this sense, our formula for $\Delta \sigma_{x x}$ is indispensable for the study of the strongly correlated Fermi liquid. Note that our formula coincides with eq. (4) if all the vertex corrections arising from $\mathcal{T}_{22}$ are neglected.

The contents of this paper are the following: In §II, we study the tight-binding model with Coulomb interaction, where the vector potential is included as the Peierls phase in the hopping integrals. In §III, the expression for the magnetoconductivity (MC) is obtained without vertex corrections, for an instructive purpose. It coincides with eq. (4⿴囗十) at lower temperatures. In $\S \mathrm{IV}$ and $\mathrm{V}$, we derive the general expression for the $\mathrm{MC}$ by taking all the vertex corrections into account, which is the main part of this work. In the former section, we derive all the vertexes corrections by taking account of the Ward identities for multi-point vertices seriously. In the later section, we perform the analytic continuation and derive the exact expression for the $\mathrm{MC}$ of order $\tau^{3}$. It is given by eq.(77) or eq.(78). Based on the obtained formula, we present several discussions on the vertex corrections, and give some remarks in §VI.

\section{KUBO FORMULA FOR THE MAGNETOCONDUCTIVITY}

\section{A. Tight Binding Model in a Magnetic Field}

The tight-binding model without the magnetic field is given by

$$
\begin{aligned}
H_{B=0} & =H_{B=0}^{0}+H_{\mathrm{int}} \\
H_{B=0}^{0} & =\sum_{\langle i, j\rangle, \sigma} t_{i, j}^{0} c_{i \sigma}^{\dagger} c_{j \sigma} \\
& =\sum_{k \sigma} \epsilon_{k}^{0} c_{k \sigma}^{\dagger} c_{k \sigma},
\end{aligned}
$$

where $t_{i, j}^{0}$ is the hopping integral and $\epsilon_{k}^{0}$ is the spectrum of the non-interacting particles, which is given by the Fourier transformation of $t_{i, j}^{0} \cdot c_{i \sigma}^{\dagger}\left(c_{k \sigma}^{\dagger}\right)$ is the creation operator of the electron at $\vec{r}_{i}$ (with momentum $\vec{k}$ ) with the spin $\sigma$. Hereafter, we may write the vectors $\vec{r}_{i}$ and $\vec{k}$ as $r$ and $k$ for simplicity. $H_{\text {int }}$ represents the two-body interaction term. In this manuscript, we assume the density-density interactions, like the on-site Coulomb interaction. Note that we exclude the processes which would change the form of the current operator, for example, the pair-hopping processes $J c_{i \uparrow}^{\dagger} c_{i \downarrow}^{\dagger} c_{j \downarrow} c_{j \uparrow}$.

Now, we introduce the magnetic field through the vector potential $\vec{A}_{i}$ on $i$-site. We do not consider the Zeeman term in this article because we focus only on the orbital effect, i.e., the Lorentz force. If the magnetic field is almost uniform, $\vec{A}_{i}$ is included in the Hamiltonian through the hopping integral as the Peierls phase factor [13]:

$$
\begin{aligned}
t_{i, j} & \equiv t_{i, j}^{0} \cdot \exp \left(-\mathrm{i} e\left(\vec{A}_{i}+\vec{A}_{j}\right) \cdot\left(\vec{r}_{i}-\vec{r}_{j}\right) / 2\right), \\
t_{j, i} & =\left\{t_{i, j}\right\}^{*}
\end{aligned}
$$

Here and hereafter, $-e(e>0)$ is the charge of an electron, and we put $c=\hbar=1$.

From now on, we introduce the external vector potential as

$$
\vec{A}_{i}^{\mathrm{tot}}=\vec{A} e^{\mathrm{i} \vec{q} \cdot \vec{r}_{i}}+\vec{A}^{\prime} e^{\mathrm{i} \vec{q}^{\prime} \cdot \vec{r}_{i}}
$$

and extract the coefficient of $B \cdot B^{\prime}=(\mathrm{i} \vec{q} \times \vec{A}) \cdot\left(\mathrm{i} \vec{q}^{\prime} \times \vec{A}^{\prime}\right)$ of the conductivity. In the final stage, we put $A=A^{\prime}$ and take the uniform limit $q=q^{\prime}=0$. This procedure was originally developed in studying the Hall conductivity in dirty metals by Fukuyama et.al. [14]. In this article, we take the $z$-axis to be the direction of the magnetic field.

Now we expand the Hamiltonian with respect to $A$ and $A^{\prime}$. For the purpose of this paper, we need the terms proportional to $A, A^{\prime}$ and $A A^{\prime}$. They are given by

$$
H_{B}=H_{B=0}+e A_{\alpha} j_{\alpha}(-q)+e A_{\beta}^{\prime} \cdot j_{\beta}\left(-q^{\prime}\right)+e^{2} A_{\alpha} A_{\beta}^{\prime} \cdot j_{\alpha \beta}\left(-q-q^{\prime}\right)+\cdots,
$$

which is exact up to $O\left(q, q^{\prime}\right)$ and $O\left(q q^{\prime}\right)$. Here and hereafter, we promise that the summation with respect to $\alpha$ or $\beta$ is taken implicitly. In eq.(8), $j_{\alpha}(p), j_{\alpha \beta}(p)$ are defined as

$$
\begin{gathered}
j_{\alpha}(p)=\sum_{k} v_{\alpha}^{0}(k) c_{k-p / 2}^{\dagger} c_{k+p / 2}, \\
j_{\alpha \beta}(p)=\sum_{k} v_{\alpha \beta}^{0}(k) c_{k-p / 2}^{\dagger} c_{k+p / 2},
\end{gathered}
$$


where $v_{\alpha}^{0}(k)=\partial_{\alpha} \epsilon_{k}^{0}$ and $v_{\alpha \beta}^{0}(k)=\partial_{\alpha \beta} \epsilon_{k}^{0}$, respectively. (Hereafter, $\partial_{\alpha} \equiv \partial / \partial k_{\alpha}$ and $\partial_{\alpha \beta} \equiv \partial^{2} / \partial k_{\alpha} \partial k_{\beta}$, etc.) The equation (8) is derived in Appendix A.

Next, we examine the current operator in the magnetic field. It is given by $\vec{j}^{B}=\vec{j}+e \vec{A} / m$ for a electron in vacuum as is well known. However, it is no more valid for a tight-binding model. By using the kinetic equation for an electron at $\vec{r}$ in the interacting representation, the current operator of the system, $\vec{j}^{B}(r)$, is given by

$$
\vec{j}^{B}(\vec{r})=\mathrm{i}\left[H_{B}^{0}, \vec{r} \rho(\vec{r})\right],
$$

where $\rho(\vec{r})$ is the density operator of the system 15. Based on this definition, $\vec{j}^{B}(p)$ in the weak magnetic field is derived in Appendix A. According to the result,

$$
\begin{aligned}
j_{\nu}^{B}(p=0) & =j_{\nu}(0)+e A_{\alpha} \cdot j_{\nu \alpha}(-q)+e A_{\beta}^{\prime} \cdot j_{\nu \alpha}\left(-q^{\prime}\right)+e^{2} A_{\alpha} A_{\beta}^{\prime} \cdot j_{\nu \alpha \beta}\left(-q-q^{\prime}\right)+\cdots, \\
j_{\nu \alpha \beta}(p) & =\sum_{k} v_{\nu \alpha \beta}^{0}(k) c_{k-p / 2}^{\dagger} c_{k+p / 2},
\end{aligned}
$$

where $v_{\nu \alpha \beta}^{0}(k) \equiv \partial_{\nu \alpha \beta} \epsilon_{k}^{0}$. Equation (12) is exact up to $O\left(q, q^{\prime}\right)$ and $O\left(q q^{\prime}\right)$. In eqs.(8) and (12), we dropped the terms proportional to $A^{2}, A^{\prime 2}$ because they are not required for the purpose of this paper. In the same way, we find

$$
j_{\mu}^{B}\left(q+q^{\prime}\right)=j_{\mu}\left(q+q^{\prime}\right)+e A_{\alpha} \cdot j_{\mu \alpha}\left(q^{\prime}\right)+e A_{\beta}^{\prime} \cdot j_{\mu \beta}(q)+e^{2} A_{\alpha} A_{\beta}^{\prime} \cdot j_{\mu \alpha \beta}(0)+\cdots,
$$

up to $O\left(A, A^{\prime}\right)$ and $O\left(A A^{\prime}\right)$.

\section{B. Kubo Formula for the Conductivity}

According to Kubo formula [16], the conductivity in the magnetic filed is given by

$$
\begin{aligned}
& \sigma_{\mu \nu}\left(q+q^{\prime}, \omega\right)=\frac{1}{\mathrm{i} \omega}\left[\Phi_{\mu \nu}\left(q+q^{\prime}, \omega+\mathrm{i} 0\right)-\Phi_{\mu \nu}\left(q+q^{\prime}, \mathrm{i} 0\right)\right], \\
& \Phi_{\mu \nu}\left(q+q^{\prime}, \omega_{\lambda}\right)=\int_{0}^{\beta} d \tau e^{-\omega_{\lambda} \tau}\left\langle T_{\tau} j_{\mu}^{B}\left(q+q^{\prime}, 0\right) j_{\nu}^{B}(0, \tau)\right\rangle_{B},
\end{aligned}
$$

where $\beta=1 / T$ and $\omega_{\lambda}=\mathrm{i} \pi T \cdot 2 \lambda$ ( $\lambda$ being the integer), respectively. Here $T_{\tau}$ is a $\tau$ ordering operator. $\Phi_{\mu \nu}\left(q+q^{\prime}, \omega+\mathrm{i} 0\right)$ in eq.(15) is given by the analytic continuation of $\Phi_{\mu \nu}\left(q+q^{\prime}, \omega_{\lambda}\right)$ in eq.(14) with respect to $\operatorname{Im} \omega_{\lambda}>0$

The diagonal conductivity $\sigma_{\mu \mu}$ does not contain the term proportional to $B^{2 n+1}$ because of the Onsager's reciprocity theorem. The diagonal conductivity given by eq.(15) can be expanded with respect to $q, q^{\prime}$ and $A, A^{\prime}$ as

$$
\sigma_{\mu \mu}\left(q+q^{\prime}, 0\right)=\sigma_{\mu \mu}^{0}+2 C_{\mu \mu}^{\alpha \rho ; \beta \rho^{\prime}} \cdot\left(\mathrm{i} q_{\rho} A_{\alpha} \cdot \mathrm{i} q_{\rho^{\prime}}^{\prime} A_{\beta}^{\prime}\right)+\cdots .
$$

The coefficient $C_{\mu \mu}^{\alpha \rho ; \beta \rho^{\prime}}$ should satisfy the relation $C_{\mu \mu}^{\alpha \rho ; \beta \rho^{\prime}}=C_{\mu \mu}^{x y ; x y} \cdot \epsilon_{z \rho \alpha} \epsilon_{z \rho^{\prime} \beta}$ because of the requirement from the gauge-invariance. We call $\Delta \sigma_{\mu \mu} \equiv C_{\mu \mu}^{\alpha \rho ; \beta \rho^{\prime}} \cdot\left(\mathrm{i} q_{\rho} A_{\alpha} \cdot \mathrm{i} q_{\rho^{\prime}}^{\prime} A_{\beta}^{\prime}\right)=C_{\mu \mu}^{x y ; x y} \cdot B^{2}$ the magnetoconductivity (MC). The aim of this paper is to derive the expression for $C_{\mu \mu}^{\alpha \rho ; \beta \rho^{\prime}}$ by using the Fermi liquid theory.

In order to derive $C_{\mu \mu}^{\alpha \rho ; \beta \rho^{\prime}}$ in eq.(16), we study the term proportional to $e^{2} A_{\alpha} A_{\beta}^{\prime}$ in eq.(15), $\Phi_{\mu \nu}^{(2)}\left(q+q^{\prime}, \omega_{\lambda}\right)$ : It is given by

$$
\begin{aligned}
\Phi_{\mu \nu}^{(2)}\left(q+q^{\prime}, \omega_{\lambda}\right)= & \int_{0}^{\beta} d \tau e^{-\omega_{\lambda} \tau}\left\{\left\langle T_{\tau} j_{\mu}\left(q+q^{\prime}, 0\right) j_{\nu \alpha \beta}\left(-q-q^{\prime}, \tau\right)\right\rangle\right. \\
& +\int_{0}^{\beta} d \tau^{\prime}\left\langle T_{\tau} j_{\mu}\left(q+q^{\prime}, 0\right) j_{\nu \alpha}(-q, \tau) j_{\beta}\left(-q^{\prime}, \tau^{\prime}\right)\right\rangle \\
& +\int_{0}^{\beta} d \tau^{\prime}\left\langle(\alpha, q) \leftrightarrow\left(\beta, q^{\prime}\right)\right\rangle \\
& +\int_{0}^{\beta} d \tau^{\prime}\left\langle T_{\tau} j_{\mu}\left(q+q^{\prime}, 0\right) j_{\nu}(0, \tau) j_{\alpha \beta}\left(-q-q^{\prime}, \tau^{\prime}\right)\right\rangle \\
& \left.+\int_{0}^{\beta} d \tau^{\prime} d \tau^{\prime \prime}\left\langle T_{\tau} j_{\mu}\left(q+q^{\prime}, 0\right) j_{\nu}(0, \tau) j_{\alpha}\left(-q, \tau^{\prime}\right) j_{\beta}\left(-q, \tau^{\prime \prime}\right)\right\rangle\right\}+\cdots
\end{aligned}
$$


(i)

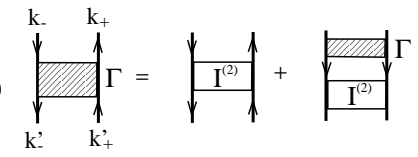

(ii)

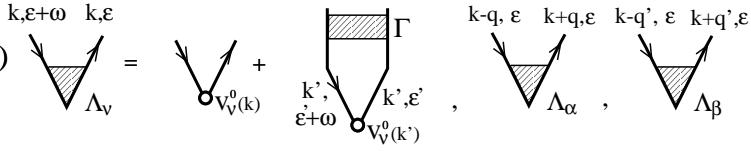

(iii)

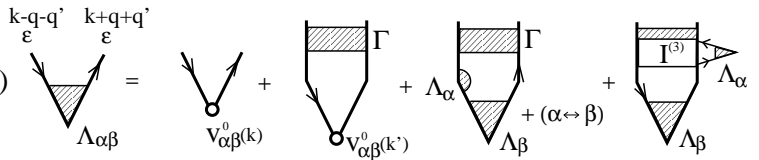

FIG. 1. (i) The Bethe-Salpeter integral equation for the four point vertex $\Gamma\left(k_{-} ; k_{+} \mid k_{+}^{\prime} ; k_{-}^{\prime}\right)$. $\Gamma^{I}$ is the irreducible vertex. (ii) Integral equations for $\Lambda_{\nu}, \Lambda_{\alpha}$ and $\Lambda_{\beta}$. (iii) The integral equation for $\Lambda_{\alpha \beta}$.

In later sections, we take the derivative of $\Phi_{\mu \nu}^{(2)}\left(q+q^{\prime}, \omega_{\lambda}\right)$ with respect to $q_{\rho}$ and $q_{\rho^{\prime}}^{\prime}$, and perform the analytic continuation with respect to $\omega_{\lambda}$. In the next stage, we derive the general formula for the $\mathrm{MC}, C_{\mu \nu}^{\alpha \rho} ; \beta \rho^{\prime}=$ $\frac{1}{2}\left(\partial^{2} / \partial q_{\rho} \partial q_{\rho^{\prime}}\right) \operatorname{Im} \Phi_{\mu \nu}^{(2)}\left(q+q^{\prime}, \omega+\mathrm{i} 0\right) /\left.\omega\right|_{\omega, q, q^{\prime} \rightarrow 0}$. Hereafter, we consider $\Phi_{\mu \nu}^{(2)}\left(2 q+2 q^{\prime}, \omega_{\lambda}\right)$ to simplify the calculations, and divide the final result by four.

In eq.(17), the terms with vector potential(s) in $j_{\mu}^{B}\left(q+q^{\prime}, 0\right)$, given by eq.(13), were dropped. Actually, it is easy to see that they make $\Phi_{\mu \nu}\left(q+q^{\prime}, \omega\right)$ independent of $q$ or $q^{\prime}$, which lead to $C_{\mu \mu}^{\alpha \rho ; \beta \rho^{\prime}}=0$

\section{Vertex Corrections}

The definition of the one particle Green function is

$$
G\left(k, \epsilon_{n}\right)=-\frac{1}{\beta} \int_{0}^{\beta} d \tau d \tau^{\prime} \epsilon^{\epsilon_{n}\left(\tau-\tau^{\prime}\right)}\left\langle T_{\tau} c_{\sigma k}(\tau) c_{\sigma k}^{\dagger}\left(\tau^{\prime}\right)\right\rangle,
$$

where $\epsilon_{n}=\mathrm{i} \pi T(2 n+1)$ is the Matsubara frequency for fermion. By introducing the self-energy $\Sigma\left(k, \epsilon_{n}\right)$, the Green function for the interacting system is expressed as

$$
G\left(k, \epsilon_{n}\right)=\left(\epsilon_{n}+\mu-\epsilon_{k}^{0}-\Sigma\left(k, \epsilon_{n}\right)\right)^{-1},
$$

where $\mu$ is the chemical potential.

As shown in Fig.1 (i), the full four-point vertex $\Gamma$ is given by the following integral equation:

$$
\begin{aligned}
\Gamma\left(k_{-} \epsilon^{+} ;\right. & \left.k_{+} \epsilon \mid k_{+}^{\prime} \epsilon^{\prime} ; k_{-}^{\prime} \epsilon^{++}\right)=\Gamma^{I}\left(k_{-} \epsilon^{+} ; k_{+} \epsilon \mid k_{+}^{\prime} \epsilon^{\prime} ; k_{-}^{\prime} \epsilon^{++}\right) \\
& +T \sum_{k^{\prime \prime} \epsilon^{\prime \prime}} \Gamma^{I}\left(k_{-} \epsilon^{+} ; k_{+} \epsilon \mid k_{+}^{\prime \prime} \epsilon^{\prime \prime} ; k_{-}^{\prime \prime} \epsilon^{\prime \prime+}\right) G\left(k_{-}^{\prime \prime}, \epsilon^{\prime \prime+}\right) G\left(k_{+}^{\prime \prime}, \epsilon^{\prime \prime}\right) \Gamma\left(k_{-}^{\prime \prime} \epsilon^{\prime \prime+} ; k_{+}^{\prime \prime} \epsilon^{\prime \prime} \mid k_{+}^{\prime} \epsilon^{\prime} ; k_{-}^{\prime} \epsilon^{\prime+}\right),
\end{aligned}
$$

where we put $k_{ \pm}=k \pm q$ and $\epsilon_{n}^{+}=\epsilon_{n}+\omega_{\lambda} . \Gamma^{I}$ is the 'irreducible' four point vertex with respect to the particle-hole pair. Here and hereafter, we do not write the spin suffix explicitly, and the suffix of the Matsubara frequencies $n$ and $\lambda$ are sometimes dropped for simplicity.

The current vertex function $\Lambda_{\nu}$ is given by

$$
\Lambda_{\nu}\left(k \epsilon^{+} ; k \epsilon\right)=v_{\nu}^{0}(k)+T \sum_{k^{\prime} \epsilon^{\prime}} \Gamma\left(k \epsilon^{+} ; k \epsilon \mid k^{\prime} \epsilon^{\prime} ; k^{\prime} \epsilon^{\prime+}\right) G\left(k^{\prime}, \epsilon^{\prime+}\right) G\left(k^{\prime}, \epsilon^{\prime}\right) v_{\nu}^{0}\left(k^{\prime}\right),
$$

as is shown in Fig.11(ii). In the same way,

$$
\Lambda_{\alpha}\left(k_{-} \epsilon ; k_{+} \epsilon\right)=v_{\alpha}^{0}(k)+T \sum_{k^{\prime} \epsilon^{\prime}} \Gamma\left(k_{-} \epsilon ; k_{+} \epsilon \mid k_{+}^{\prime} \epsilon^{\prime} ; k_{-}^{\prime} \epsilon^{\prime}\right) G\left(k_{+}^{\prime}, \epsilon^{\prime}\right) G\left(k_{-}^{\prime}, \epsilon^{\prime}\right) v_{\mu}^{0}\left(k^{\prime}\right) .
$$




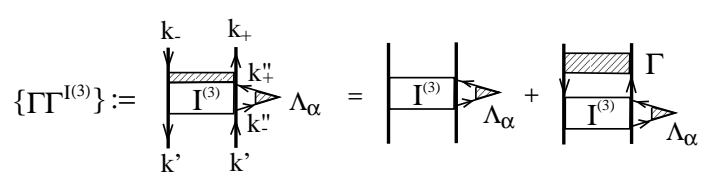

FIG. 2. The definition of $\left\{\Gamma \Gamma^{I(3)}\right\}\left(k_{-} ; k_{+}\left|k_{+}^{\prime \prime} ; k_{-}^{\prime \prime}\right| k^{\prime} ; k^{\prime}\right)$.

We also introduce the vertex $\Lambda_{\alpha \beta}$ which is related to the bare vertex $v_{\alpha \beta}^{0}(k)=\partial_{\alpha \beta} \epsilon_{k}^{0}$ as follows:

$$
\begin{aligned}
& \Lambda_{\alpha \beta}\left(k_{--} \epsilon ; k_{++} \epsilon\right)=v_{\alpha \beta}^{0}(k)+T \sum_{k^{\prime} \epsilon^{\prime}} \Gamma\left(k_{--} ; k_{++} \mid k_{++}^{\prime} ; k_{--}^{\prime}\right) \cdot G\left(k_{--}^{\prime}\right) G\left(k_{++}^{\prime}\right) \\
& \quad \times\left(v_{\alpha \beta}^{0}\left(k^{\prime}\right)+\Lambda_{\alpha}\left(k_{--}^{\prime}, k_{+-}^{\prime}\right) G\left(k_{+-}^{\prime}\right) \Lambda_{\beta}\left(k_{+-}^{\prime} ; k_{++}^{\prime}\right)+\Lambda_{\beta}\left(k_{--}^{\prime} ; k_{-+}^{\prime}\right) G\left(k_{-+}^{\prime}\right) \Lambda_{\alpha}\left(k_{-+}^{\prime} ; k_{++}^{\prime}\right)\right) \\
& \quad T^{2} \sum_{k^{\prime} k^{\prime \prime} \epsilon^{\prime} \epsilon^{\prime \prime}}\left\{\Gamma \Gamma^{I(3)}\right\}\left(k_{--} ; k_{++}\left|k_{+}^{\prime} ; k_{-}^{\prime}\right| k_{0+}^{\prime \prime} ; k_{0-}^{\prime \prime}\right) \cdot G\left(k_{-}^{\prime}\right) \Lambda_{\alpha}\left(k_{-}^{\prime} ; k_{+}^{\prime}\right) G\left(k_{+}^{\prime}\right) \cdot G\left(k_{0-}^{\prime \prime}\right) \Lambda_{\beta}\left(k_{0-}^{\prime \prime} ; k_{0+}^{\prime \prime}\right) G\left(k_{0+}^{\prime \prime}\right),
\end{aligned}
$$

where $k_{ \pm \pm} \equiv k \pm q \pm q^{\prime}, k_{ \pm \mp} \equiv k \pm q \mp q^{\prime}$ and $k_{0 \pm} \equiv k \pm q^{\prime}$, respectively. (Here frequency variables were dropped because it would not cause a confusion in that case.) The eq.(23) is expressed diagrammatically in Fig.1 (iii). Here, $\Gamma^{I(3)}$ is the irreducible six-point vertex, and the vertex $\left\{\Gamma \Gamma^{I(3)}\right\}$ is defined as

$$
\begin{aligned}
& \left\{\Gamma \Gamma^{I(3)}\right\}\left(k_{--} ; k_{++}\left|k_{+}^{\prime} ; k_{-}^{\prime}\right| k_{0+}^{\prime \prime} ; k_{0-}^{\prime \prime}\right) \\
& \quad=T \sum_{p \epsilon_{m}}\left(\delta_{k, p} / T+\Gamma\left(k_{--} ; k_{++} \mid p_{++} ; p_{--}\right) G\left(p_{--}\right) G\left(p_{++}\right)\right) \Gamma^{I(3)}\left(p_{--} ; p_{++}\left|k_{+}^{\prime} ; k_{-}^{\prime}\right| k_{0+}^{\prime \prime} ; k_{0-}^{\prime \prime}\right),
\end{aligned}
$$

which is also depicted in Fig. 22.

In the case of $q=0$, Ward identity assures the following relations [17, 18$]$ :

$$
\begin{aligned}
\Lambda_{\alpha}\left(k \epsilon_{n} ; k \epsilon_{n}\right) & =v_{\alpha}^{0}(k)+\partial_{\alpha} \Sigma\left(k, \epsilon_{n}\right), \\
\Lambda_{\alpha \beta}\left(k \epsilon_{n} ; k \epsilon_{n}\right) & =v_{\alpha \beta}^{0}(k)+\partial_{\alpha \beta} \Sigma\left(k, \epsilon_{n}\right) .
\end{aligned}
$$

Now we analyze $\Phi_{\mu \nu}^{(2)}\left(2 q+2 q^{\prime}, \omega_{\lambda}\right)$ in terms of the diagrammatic technique. They are expressed in Figs. 3 and 4 . Below, we show analytical expressions for some of them:

$$
\begin{aligned}
(\mathrm{a})= & -T \sum_{k, \epsilon} \Lambda_{\mu}\left(k_{++} ; k_{--}\right) G^{+}\left(k_{--}\right) v_{\nu \alpha \beta}^{0}(k) G\left(k_{++}\right) \\
(\mathrm{b}-1)= & -T \sum_{k, \epsilon} \Lambda_{\mu}\left(k_{++} ; k_{--}\right) G^{+}\left(k_{--}\right) \Lambda_{\nu}\left(k_{--} ; k_{--}\right) G\left(k_{--}\right) \Lambda_{\alpha \beta}\left(k_{--} ; k_{++}\right) G\left(k_{++}\right) \\
(\mathrm{d})= & -T \sum_{k, \epsilon} \Lambda_{\mu}\left(k_{++} ; k_{--}\right) G^{+}\left(k_{--}\right) \Lambda_{\alpha}^{+}\left(k_{--} ; k_{+-}\right) G^{+}\left(k_{+-}\right) \\
& \times \Lambda_{\nu}\left(k_{+-} ; k_{+-}\right) G\left(k_{+-}\right) \Lambda_{\beta}\left(k_{+-} ; k_{++}\right) G\left(k_{++}\right)
\end{aligned}
$$

where $v_{\nu \alpha \beta}^{0} \equiv \partial_{\nu \alpha \beta} \epsilon_{k}^{0}$, and we write $G^{+}(k) \equiv G\left(k, \epsilon^{+}\right)$and $\Lambda_{\alpha}^{+}\left(k_{-} ; k_{+}\right) \equiv \Lambda_{\alpha}\left(k_{-} \epsilon^{+} ; k_{+} \epsilon^{+}\right)$, respectively. We also give expressions for some terms in Fig. 4, which include four, six and eight point vertices:

$$
\begin{aligned}
(\mathrm{f}-1)= & -T^{3} \sum_{k k^{\prime} k^{\prime \prime}, \epsilon \epsilon^{\prime} \epsilon^{\prime \prime}} \Gamma^{I(3)}\left(k_{--} \epsilon^{+} ; k_{++} \epsilon\left|k^{\prime} \epsilon^{\prime} ; k^{\prime} \epsilon^{\prime+}\right| k_{++}^{\prime \prime} \epsilon^{\prime \prime}, k_{--}^{\prime \prime} \epsilon^{\prime \prime}\right) \\
& \times G\left(k_{++}\right) \Lambda_{\mu}\left(k_{++} ; k_{--}\right) G^{+}\left(k_{--}\right) \cdot G^{+}\left(k^{\prime}\right) \Lambda_{\nu}\left(k^{\prime} ; k^{\prime}\right) G\left(k^{\prime}\right) \\
& \times G\left(k_{--}^{\prime \prime}\right) \Lambda_{\alpha \beta}\left(k_{--}^{\prime \prime} ; k_{++}^{\prime \prime}\right) G\left(k_{++}^{\prime \prime}\right), \\
(\mathrm{g}-1)= & -T^{2} \sum_{k k^{\prime}, \epsilon \epsilon^{\prime}} \Gamma\left(k_{--} \epsilon^{+} ; k_{++} \epsilon \mid k_{0+}^{\prime} \epsilon^{\prime} ; k_{0-}^{\prime} \epsilon^{\prime+}\right) \\
& \times G\left(k_{-+}\right) \Lambda_{\alpha}\left(k_{-+} ; k_{++}\right) G\left(k_{++}\right) \Lambda_{\mu}\left(k_{++} ; k_{--}\right) G^{+}\left(k_{--}\right) \\
& \times G^{+}\left(k_{0-}^{\prime}\right) \Lambda_{\nu}\left(k_{0-}^{\prime} ; k_{0-}^{\prime}\right) G\left(k_{0-}^{\prime}\right) \Lambda_{\beta}\left(k_{0-}^{\prime} ; k_{0+}^{\prime}\right) G\left(k_{0+}^{\prime}\right), \\
(\mathrm{h}-1)= & -T^{3} \sum_{k k^{\prime} k^{\prime \prime}, \epsilon \epsilon^{\prime} \epsilon^{\prime \prime}}\left\{\Gamma \Gamma^{I(3)}\right\}\left(k_{+-} \epsilon^{+} ; k_{++} \epsilon\left|k^{\prime} \epsilon^{\prime} ; k^{\prime} \epsilon^{\prime+}\right| k_{0+}^{\prime \prime} \epsilon^{\prime \prime} ; k_{0-}^{\prime \prime} \epsilon^{\prime \prime}\right)
\end{aligned}
$$




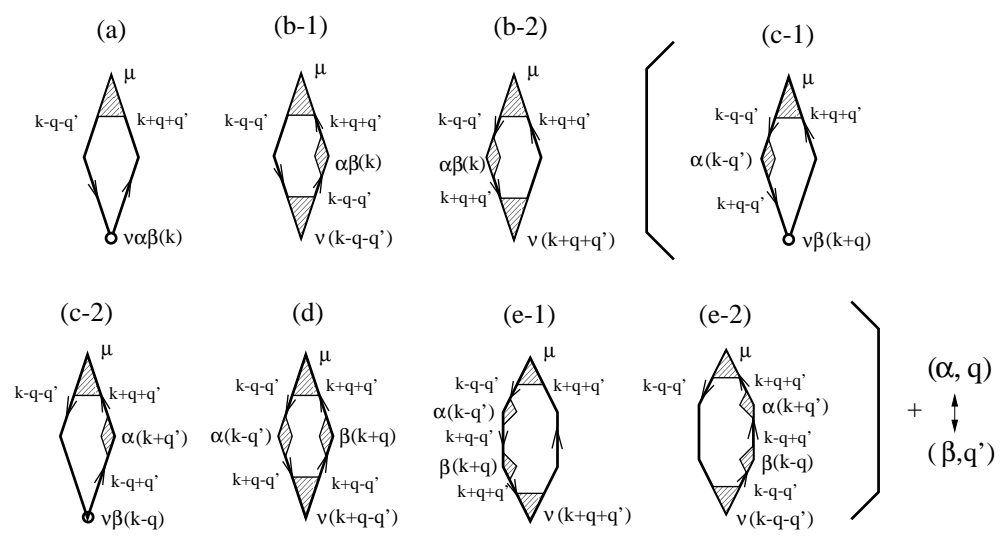

FIG. 3. The expressions for $\Phi_{\mu \nu}^{(2)}\left(2 q+2 q^{\prime}, \omega_{\lambda}\right)$, which are composed of $\vec{\Lambda}, G$ and $\vec{v}^{0}$.
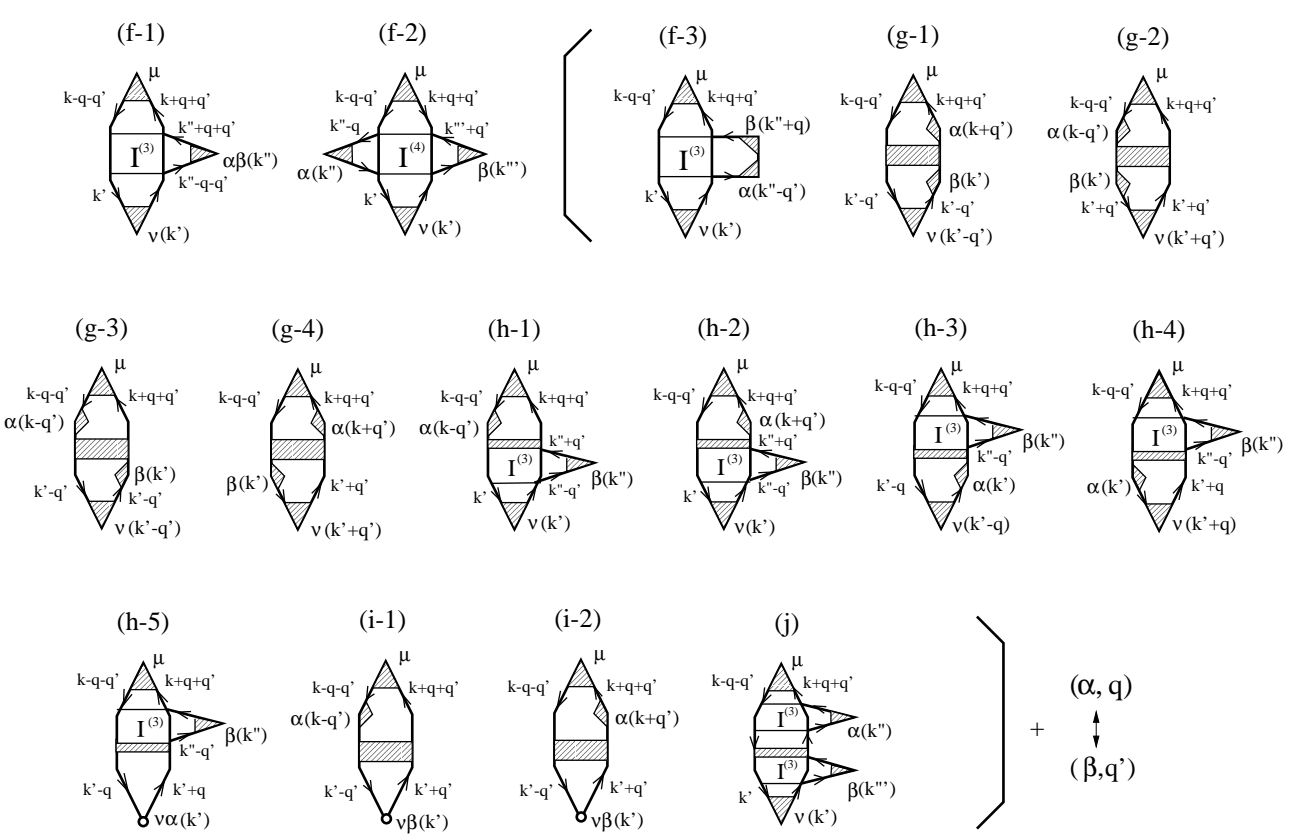

FIG. 4. The expressions for $\Phi_{\mu \nu}^{(2)}\left(2 q+2 q^{\prime}, \omega_{\lambda}\right)$, which contains at least one four (or six, eight) point vertex. 


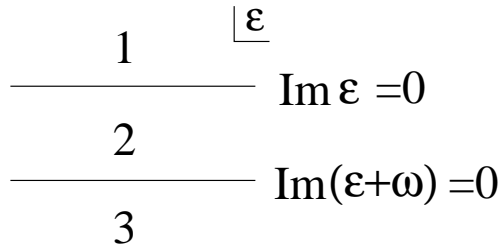

FIG. 5. The analytic regions $1 \sim 3$ as a function of a complex variable $\epsilon$ (or $\epsilon^{\prime}$ ). Here we put $\operatorname{Im} \omega_{\lambda}>0$. From each region, $g\left(\epsilon_{n} ; \omega_{\lambda}\right), \Lambda_{\mu}\left(\epsilon_{n}^{+} ; \epsilon_{n}\right)$ and $\Gamma\left(\epsilon_{n}^{+} ; \epsilon_{n} \mid \epsilon_{n^{\prime}}^{+} ; \epsilon_{n^{\prime}}\right)$ are analytically continued to be $g_{i}(\epsilon ; \omega), J_{\mu}^{(i)}\left(\epsilon^{+} ; \epsilon\right)$ and $\mathcal{T}_{i j}\left(\epsilon^{+} ; \epsilon \mid \epsilon^{\prime} ; \epsilon^{\prime+}\right)(i, j=1 \sim 3)$, respectively.

$$
\begin{aligned}
& \times G\left(k_{++}\right) \Lambda_{\mu}\left(k_{++} ; k_{--}\right) G^{+}\left(k_{--}\right) \Lambda_{\alpha}^{+}\left(k_{--} ; k_{+-}\right) G^{+}\left(k_{+-}\right) \\
& \times G^{+}\left(k^{\prime}\right) \Lambda_{\nu}\left(k^{\prime} ; k^{\prime}\right) G\left(k^{\prime}\right) \cdot G\left(k_{0-}^{\prime \prime}\right) \Lambda_{\beta}\left(k_{0-}^{\prime \prime} ; k_{0+}^{\prime \prime}\right) G\left(k_{0+}^{\prime \prime}\right),
\end{aligned}
$$

where $\left\{\Gamma \Gamma^{I(3)}\right\}$ is defined in eq. (24).

\section{MAGNETOCONDUCTIVITY: WITHOUT VERTEX CORRECTIONS}

In this section, we derive the MC by neglecting all the current vertex corrections in the first place. We will see that this approximation gives the consistent result with the relaxation time approximation (RTA). In later sections, we derive the exact formula for the $\mathrm{MC}$ of order $\gamma^{-3}$, by taking all the current vertex corrections into account.

\section{A. analytic continuation}

In deriving the conductivity, we have to perform the analytic continuation of $\Phi_{\mu \nu}^{(2)}\left(2 q+2 q^{\prime}, \omega_{\lambda}\right)$. For an instructive purpose, we briefly review the conductivity $\sigma_{\mu \nu}$ without the magnetic field. If we neglect all the vertex corrections for the current, the current-current correlation without the magnetic filed, $\Phi_{\mu \nu}^{(0)}(\omega)$, is simply given by

$$
\Phi_{\mu \nu}^{(0)}\left(\omega_{\lambda}\right)=-T \sum_{k \epsilon_{n}} G\left(k, \epsilon_{n}^{+}\right) G\left(k, \epsilon_{n}\right) \cdot v_{\mu}^{0}(k) v_{\nu}^{0}(k),
$$

where $\epsilon_{n}^{+}=\epsilon_{n}+\omega_{\lambda}$. After the analytic continuation with respect to $\operatorname{Im} \omega_{\lambda}>0$, we get [9]

$$
\Phi_{\mu \nu}^{(0)}(\omega)=-2 \sum_{k} \int \frac{d \epsilon}{4 \mathrm{i} \pi}\left[\operatorname{th} \frac{\epsilon}{2 T} g_{1}(k, \epsilon ; \omega)+\left(\operatorname{th} \frac{\epsilon^{+}}{2 T}-\operatorname{th} \frac{\epsilon}{2 T}\right) g_{2}(k, \epsilon ; \omega)-\operatorname{th} \frac{\epsilon^{+}}{2 T} g_{3}(k, \epsilon ; \omega)\right] v_{\mu}^{0}(k) v_{\nu}^{0}(k),
$$

where $\epsilon^{+}=\epsilon+\omega$, and $g_{1}(k, \epsilon ; \omega)=G^{R}\left(k, \epsilon^{+}\right) G^{R}(k, \epsilon), g_{2}(k, \epsilon ; \omega)=G^{R}\left(k, \epsilon^{+}\right) G^{A}(k, \epsilon)$ and $g_{3}(k, \epsilon ; \omega)=$ $G^{A}\left(k, \epsilon^{+}\right) G^{A}(k, \epsilon)$, respectively.

Here $G^{R}(\epsilon)=G(\epsilon+\mathrm{i} 0)\left(G^{A}(\epsilon)=G(\epsilon-\mathrm{i} 0)\right)$ is the retarded (advanced) function. In eq.(34), the first, second and third terms come from the region 1, 2 and 3 in Fig. 5, respectively. The coefficient 2 in front of the $k$-summation in eq.(34) comes from the spin-degeneracy. Hereafter, we take this factor 2 correctly in all the expressions in this article.

In a Fermi liquid, $G^{R}(k, \epsilon)$ is expressed for $\epsilon \sim 0$ and $|\vec{k}| \sim k_{\mathrm{FS}}$ as

$$
G^{R}(k, \epsilon)=z_{k}\left(\epsilon+\mu-\epsilon_{k}^{*}+\mathrm{i} \gamma_{k}^{*}\right)^{-1},
$$

where $z_{k}=\left.(1-(\partial / \partial \epsilon) \operatorname{Re} \Sigma(k, \epsilon))^{-1}\right|_{\epsilon=0}$ is the renormalization factor at the Fermi energy. We also introduced the quantities $\epsilon_{k}=\epsilon_{k}^{0}+\operatorname{Re} \Sigma(k, 0), \gamma_{k}=-\operatorname{Im} \Sigma^{R}(k, 0)>0$, and their 'renormalized quantities' $\epsilon_{k}^{*}=z_{k}\left(\epsilon_{k}-\mu\right)+\mu$ and $\gamma_{k}^{*}=z_{k} \gamma_{k}$, respectively.

At sufficiently low temperatures, $\gamma_{k}^{*} \ll T$ is satisfied in a Fermi liquid because $\gamma_{k}^{*} \propto T^{2}$ in $3 \mathrm{D}$ and $\gamma_{k}^{*} \propto T^{2} \log T^{-1}$ in $2 \mathrm{D}$. In this case,

$$
\begin{aligned}
& g_{2}(k, \epsilon ; 0) \approx \pi z_{k} \delta\left(\epsilon+\mu-\epsilon_{k}^{*}\right) / \gamma_{k}, \\
& g_{1}(k, \epsilon ; 0) \approx\left(\frac{z_{k}}{\epsilon+\mu-\epsilon_{k}^{*}+\mathrm{i} \delta}\right)^{2} .
\end{aligned}
$$


After the integration with respect to $\epsilon$, eq.(36) gives the singular term of order $\gamma^{-1} \sim \tau^{1}$. Whereas eq.(37), which have no pole in the region $\operatorname{Im} \epsilon \geq 0$, give no singularities after the $\epsilon$-integration.

In conclusion, the conductivity $\sigma_{\mu \nu}=\lim _{\omega \rightarrow 0} \operatorname{Im} \Phi_{\mu \nu}(\omega) / \omega$ comes from the term possessing $g_{2}$-section in eq. (34) as follows:

$$
\sigma_{\mu \nu}=e^{2} \sum_{k}\left(-\frac{\partial f}{\partial \epsilon}\right)_{\epsilon_{k}^{*}-\mu} z_{k} \cdot \frac{1}{\gamma_{k}} v_{\mu}^{0}(k) v_{\nu}^{0}(k)
$$

which is proportional to $\gamma^{-1}$. Note that we get the exact expression (2) if we take account of all the current vertex correction completely. It is still of order $\gamma^{-1}$ because $J_{k}$ is of order $\gamma^{0}$. In the next subsection, we perform the analytic continuation for the $\mathrm{MC}$ in a similar way, and we also see that only the term which comes from the region 2 in Fig. 目 gives the most singular contribution with respect to $\gamma^{-1}$.

\section{B. Magnetoconductivity}

In this subsection, we perform the analytic continuation and derive the expression for the $\mathrm{MC}$ without vertex corrections for the current. In this approximation, (i) all the diagrams in Fig. A are dropped because each of them contains more than one four (or six, eight) point vertices, and (ii) the three-point vertices $\Lambda_{\mu(\nu)}$ in Fig 3 are replaced with the bare ones, $v_{\mu(\nu)}^{0}(k)$. On the other hand, we notice the Ward identities for $\Lambda_{\alpha}$ and $\Lambda_{\alpha \beta}$ given by eqs. (25) and (26), in order to get the gauge-invariant expression for the MR as shown below.

As explained in the previous subsection, the most singular terms with respect to $\gamma^{-1}$ come from the region 2 in Fig. 5. Thus, we replace $G\left(k, \epsilon^{+}\right) \rightarrow G^{R}(k, \epsilon)$ and $G(k, \epsilon) \rightarrow G^{A}(k, \epsilon)$, respectively. In this subsection, we take the $q_{\rho}, q_{\rho^{\prime}}^{\prime}$-derivative of $\Phi^{(2)}\left(2 q+2 q^{\prime}, \omega_{\lambda}\right)$ in order to derive the MC. For this purpose, we use the relation

$$
\begin{array}{r}
\Lambda_{\alpha}^{R}\left(k_{-} \epsilon ; k_{+} \epsilon\right)=V_{\alpha}^{R}(k, \epsilon)+O\left(q^{2}\right), \\
V_{\alpha}^{R}(k, \epsilon) \equiv v_{\alpha}^{0}(k)+\partial_{\alpha} \Sigma(k, \epsilon+\mathrm{i} \delta) \\
=v_{\alpha}(k, \epsilon)-\mathrm{i} \gamma_{\alpha}(k, \epsilon),
\end{array}
$$

where $v_{\alpha}(k, \epsilon)=v_{\alpha}^{0}(k)+\partial_{\alpha} \operatorname{Re} \Sigma(k, \epsilon)$ and $\gamma_{\alpha}(k, \epsilon)=-\partial_{\alpha} \operatorname{Im} \Sigma(k, \epsilon+\mathrm{i} \delta)$. They are shown in Fig.6 (i). As a result, $\omega$-linear term of $\Phi^{(2)}\left(2 q+2 q^{\prime}, \omega+\mathrm{i} 0\right)$ without the vertex corrections is given by

$$
\begin{aligned}
& \lim _{\omega \rightarrow 0} \Phi^{(2)}\left(2 q+2 q^{\prime}, \omega+\mathrm{i} 0\right) / \omega \\
&=- \sum_{k} \int \frac{d \epsilon}{\pi}\left(-\frac{\partial f}{\partial \epsilon}\right) \cdot v_{\mu}^{0}(k) G^{R}\left(k_{--}\right) G^{A}\left(k_{++}\right) \\
& \times\left\{v_{\nu \alpha \beta}^{0}(k)+\left(V_{\alpha \beta}^{R}(k) G^{R}\left(k_{++}\right) v_{\nu}^{0}\left(k_{++}\right)+v_{\nu}^{0}\left(k_{--}\right) G^{A}\left(k_{--}\right) V_{\alpha \beta}^{A}(k)\right)\right. \\
&+ {\left[\left(V_{\alpha}^{R}\left(k_{0-}\right) G^{R}\left(k_{+-}\right) v_{\nu \beta}^{0}\left(k_{+}\right)+v_{\nu \beta}^{0}\left(k_{-}\right) G^{A}\left(k_{-+}\right) V_{\alpha}^{A}\left(k_{0+}\right)\right)\right.} \\
&+V_{\alpha}^{R}\left(k_{0-}\right) G^{R}\left(k_{+-}\right) v_{\nu}^{0}\left(k_{+-}\right) G^{A}\left(k_{+-}\right) V_{\beta}^{A}\left(k_{+}\right) \\
&\left.+\left(V_{\alpha}^{R}\left(k_{0-}\right) G^{R}\left(k_{+-}\right) V_{\beta}^{R}\left(k_{+}\right) G^{R}\left(k_{++}\right) v_{\nu}^{0}\left(k_{++}\right)+v_{\nu}^{0}\left(k_{--}\right) G^{R}\left(k_{--}\right) V_{\alpha}^{R}\left(k_{0-}\right) G^{A}\left(k_{+-}\right) V_{\beta}^{A}\left(k_{+}\right)\right)\right] \\
&+ {\left.\left[(\alpha, q) \leftrightarrow\left(\beta, q^{\prime}\right)\right]\right\} . }
\end{aligned}
$$

To take the $q, q^{\prime}$-derivative of eq.(41), we employ the diagrammatic technique for the preparation of deriving the exact formula in the next section. Then, we use the followings:

$$
\begin{aligned}
& G^{R}\left(k_{ \pm}, \epsilon\right)=G^{R}(k, \epsilon) \pm q_{\rho} \cdot V_{\rho}^{R}(k, \epsilon) G^{R}(k, \epsilon)^{2}+O\left(q^{2}\right), \\
& V_{\beta}^{R}\left(k_{ \pm}, \epsilon\right)={ }_{\beta}^{R}(k, \epsilon) \pm q_{\rho} \cdot V_{\beta \rho}^{R}(k, \epsilon)+O\left(q^{2}\right),
\end{aligned}
$$

where $V_{\alpha \beta}^{R}(k, \epsilon) \equiv \partial_{\beta} V_{\alpha}^{R}(k, \epsilon)=\partial_{\alpha} V_{\beta}^{R}(k, \epsilon)$. There, we took the summations of $\rho$ and $\rho^{\prime}$ implicitly. Eq.(42) is shown in Fig.6 (ii).

Figure 7 shows the diagrammatic expressions for the $q, q^{\prime}$-derivative of (a) and (c-1) in Fig. 3. The $q$-derivative of the Green functions increases the number of $G$ 's by one, which increases the singularity of the conductivity by $\gamma^{-1}$. 
(i)

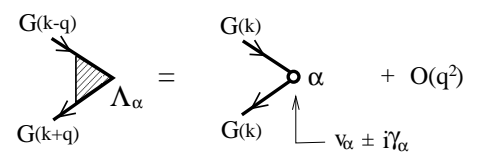

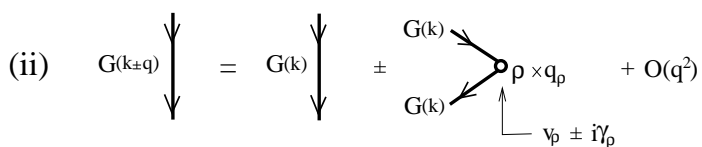

FIG. 6. The expansion of (i) $\Lambda_{\alpha}\left(k_{-} \epsilon ; k_{+} \epsilon\right)$, and (ii) $G\left(k_{ \pm}\right)$with respect to $q$.
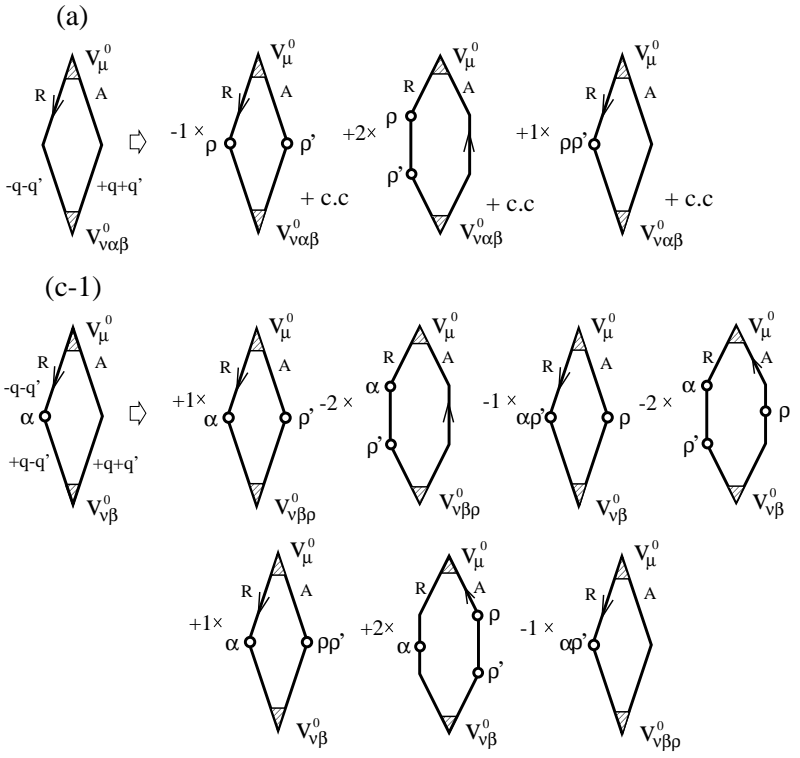

FIG. 7. The $q, q^{\prime}$-derivative of (a) and (c-1) in Fig. 3 without vertex corrections. 'R' ('A') represent retarded (advanced) Green functions. 'o $\rho$ ' on retarded (advanced) Green functions means $v_{\rho}-\mathrm{i} \gamma_{\rho}\left(v_{\rho}+\mathrm{i} \gamma_{\rho}\right)$. Note that the sign of each diagrams comes from $\partial / \partial q$ and $\partial / \partial q^{\prime}$. 


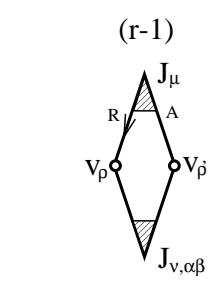

$(-2)$

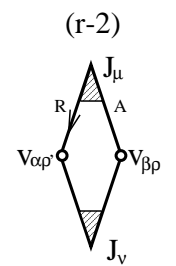

$(-1)$

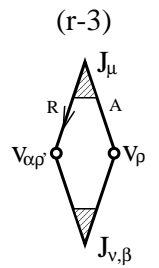

$(-2)$

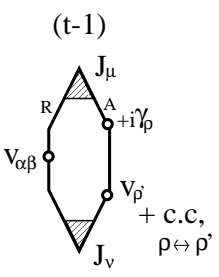

(+2) (t-2)

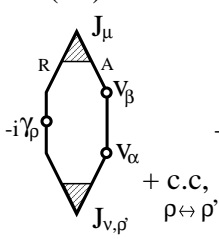

(+4)

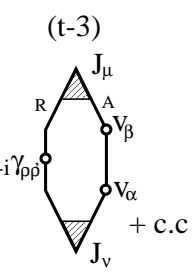

(+2)

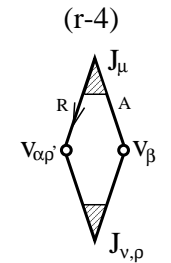

(-2)

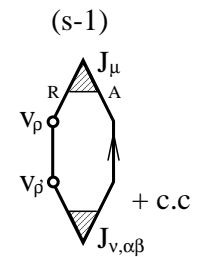

$(+2)$ (s-2)

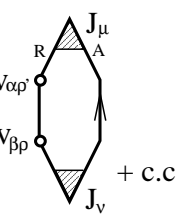

(-1)

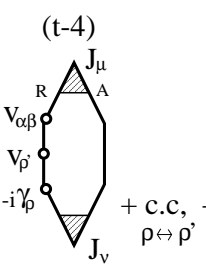

(+2)

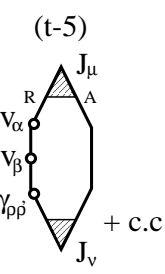

$(+2)$ $(\mathrm{t}-6)$

$+(\alpha \leftrightarrow \rho),\left(\beta \leftrightarrow \rho^{\prime}\right) \quad$ with sign change

FIG. 8 .

The diagrams for the MC of order $\gamma^{-3}$ which are composed of only $G, \vec{v}$ and $\vec{J}$. (r-1 4) corresponds to $A^{(1)}$ in eq. 44 ). In the same way, (s-1,2) corresponds to $A^{(2)},(\mathrm{t}-1 \sim 3)$ corresponds to $A^{(3)},(\mathrm{t}-4,5)$ corresponds to $A^{(4)}$, and (t-6) corresponds to $A^{(6)}$, respectively. $(\mathrm{r}-1) \sim(\mathrm{s}-2)$ contain neither $\gamma_{\alpha}$ nor $\gamma_{\alpha \beta}$, but $(\mathrm{t}-1) \sim(\mathrm{t}-6)$ contain $\gamma_{\alpha}$ and $/$ or $\gamma_{\alpha \beta}$.

In Fig. 7, the coefficient of a diagram and that of a diagram which differ by $\alpha \leftrightarrow \rho$ are opposite in sign. This fact ensures the gauge-invariance of the expression.

To derive the formula for $\mathrm{MC}$, we take the $q, q^{\prime}$-derivative of the all other terms eq.(41). Figure 8 shows the obtained terms for the MC of order $\gamma^{-3}$, which are the most singular terms as we will see later. Note that the terms $(\mathrm{t}-1 \sim 6)$ become zero in an isotropic system because they contain the momentum derivative of $\operatorname{Im} \Sigma_{k}$ along the Fermi surface. [see eq.(46) in this paper.] However, they will play important roles in rather anisotropic system like high- $T_{\mathrm{c}}$ superconductors.

In Fig. 8, we have dropped some diagrams which cancel out exactly with their counterparts. We show the example of the cancellation in Fig. 9. Also, the less singular terms with respect to $\gamma^{-1}$, some examples are shown in Fig. 10 (i), are discarded. Furthermore, we also dropped the terms proportional to $|G(k, \epsilon)|^{m} \cdot \operatorname{Im} G^{2 n}(k, \epsilon)$ or to $|G(k, \epsilon)|^{m} \cdot \operatorname{Re} G^{2 n+1}(k, \epsilon)$ : Some of the them are shown in Fig. 10 (ii). Because they are odd-functions of $\left(\epsilon+\mu-\epsilon_{k}^{*}\right)$, their contribution for the MC should be vanishingly small after the $\epsilon$ and $k$-integrations, except that the density of states is extraordinarily asymmetric around the chemical potential.

In this paper, we assume that the magnetic filed is along the $z$-axis. We can verify that the obtained expression for the MC, which is given in Fig. 8, changes its sign with the replacement $\alpha \leftrightarrow \rho$, or $\beta \leftrightarrow \rho^{\prime}$. Thus, the gauge-invariant result of the $\mathrm{MC}, C_{\mu \nu}^{\alpha \rho ; \beta \rho^{\prime}}=C_{\mu \nu}^{x y ; x y} \cdot \epsilon_{\alpha \rho z} \epsilon_{\beta \rho^{\prime} z}$ in eq.(16), is assured.

Below, we give the analytical expression for Fig. 8 .

$$
\begin{aligned}
\Delta \sigma_{\mu \nu}^{a}= & B^{2} \cdot \frac{e^{4}}{8 v_{B}} \sum_{k, i=1, \cdots, 5} \int \frac{d \epsilon}{\pi}\left(-\frac{\partial f}{\partial \epsilon}\right) J_{\mu}(k, \epsilon) \cdot A^{(i)}(k, \epsilon), \\
A^{(1)}(k, \epsilon)= & |G(k, \epsilon)|^{4} \cdot 2\left[\left(v_{x y}^{2}-v_{x x} v_{y y}\right) J_{\nu}+2\left(v_{y} v_{x y}-v_{x} v_{y y}\right) J_{\nu, x}+2\left(v_{x} v_{x y}-v_{y} v_{x x}\right) J_{\nu, y}\right. \\
& \left.+\left(v_{y}^{2} J_{\nu, x x}-2 v_{x} v_{y} J_{\nu, x y}+v_{x}^{2} J_{\nu, y y}\right)\right]_{(k, \epsilon)}, \\
A^{(2)}(k, \epsilon)= & |G(k, \epsilon)|^{2} \operatorname{Re}\left\{G_{k}^{2}(\epsilon)\right\} \cdot 4\left[\left(v_{x y}^{2}-v_{x x} v_{y y}\right) J_{\nu}-\left(v_{y}^{2} J_{\nu, x x}-2 v_{x} v_{y} J_{\nu, x y}+v_{x}^{2} J_{\nu, y y}\right)\right]_{(k, \epsilon)}, \\
A^{(3)}(k, \epsilon)= & -|G(k, \epsilon)|^{4} \operatorname{Im}\left\{G^{\mathrm{R}}(k, \epsilon)\right\} \cdot 4\left[2\left(v_{x} v_{y y} \gamma_{x}+v_{y} v_{x x} \gamma_{y}-v_{x} v_{x y} \gamma_{y}-v_{y} v_{x y} \gamma_{x}\right) J_{\nu}\right. \\
& +\left(-v_{x}^{2} \gamma_{y y}+2 v_{x} v_{y} \gamma_{x y}-v_{y}^{2} \gamma_{x x}\right) J_{\nu} \\
& \left.+4\left(v_{x} v_{y} \gamma_{y}-v_{y}^{2} \gamma_{x}\right) J_{\nu, x}+4\left(v_{x} v_{y} \gamma_{x}-v_{x}^{2} \gamma_{y}\right) J_{\nu, y}\right]_{(k, \epsilon)}, \\
A^{(4)}(k, \epsilon)= & -|G(k, \epsilon)|^{2} \operatorname{Im}\left\{G^{\mathrm{R}}(k, \epsilon)\right\} \cdot 4\left[2\left(v_{x} v_{y y} \gamma_{x}+v_{y} v_{x x} \gamma_{y}-v_{x} v_{x y} \gamma_{y}-v_{y} v_{x y} \gamma_{x}\right) J_{\nu}\right. \\
& \left.+\left(v_{x}^{2} \gamma_{y y}-2 v_{x} v_{y} \gamma_{x y}+v_{y}^{2} \gamma_{x x}\right) J_{\nu}\right]_{(k, \epsilon)},
\end{aligned}
$$



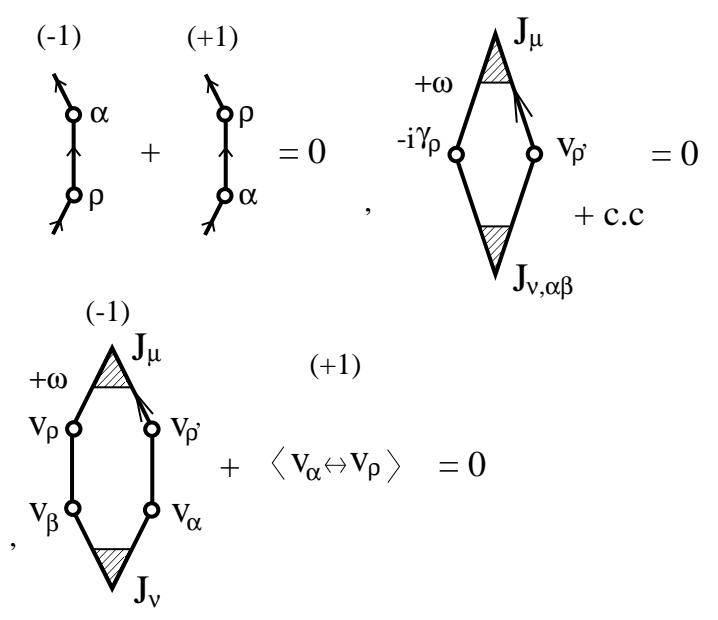

FIG. 9. Examples of the diagrams which vanish identically.

(i)
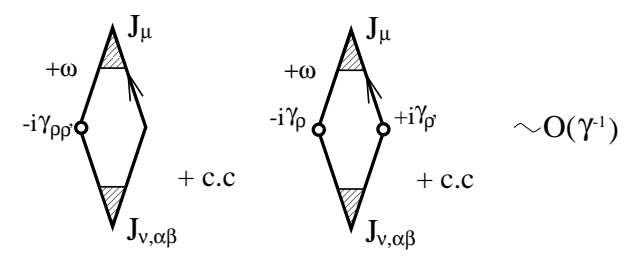

(ii)
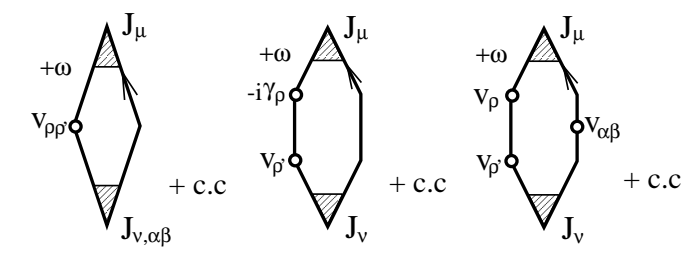

FIG. 10. (i) The diagrams of order $\gamma^{-1}$, which are negligible compared with the terms $O\left(\gamma^{-3}\right)$. (ii) The diagrams which are odd with respect to $\left(\epsilon+\mu-\epsilon_{k}^{*}\right)$ : They will be vanishingly small after the integrations with respect to $\epsilon$ and $\vec{k}$. 


$$
A^{(5)}(k, \epsilon)=|G(k, \epsilon)|^{6} \cdot 16\left[\left(v_{x}^{2} \gamma_{y}^{2}+v_{y}^{2} \gamma_{x}^{2}-2 v_{x} v_{y} \gamma_{x} \gamma_{y}\right) J_{\nu}\right]_{(k, \epsilon)}
$$

where $v_{x y} \equiv \partial_{x} v_{y}=\partial_{x y}\left(\epsilon_{k}^{0}+\operatorname{Re} \Sigma_{k}(0)\right)$ and $\gamma_{x y} \equiv \partial_{x} \gamma_{y}=\partial_{x y} \operatorname{Im} \Sigma_{k}(-\mathrm{i} \delta)$. In eq. (44), we replaced $v_{\mu(\nu)}^{0}(k)$ in eq. (41) with $J_{\mu(\nu)}(k, \epsilon)=v_{\mu(\nu)}(k, \epsilon)$, and $J_{\nu, x} \equiv v_{\nu x}(k, \epsilon), J_{\nu, x y} \equiv v_{\nu x y}(k, \epsilon)$. Note that we have checked eq.(44) by taking the $q, q^{\prime}$-derivative of eq. (41) directly by using MATHEMATICA. In later sections, we study all the vertex corrections which are dropped here, and find that each $J_{\mu}$ and $J_{\nu}$ in eq.(44) have a vertex correction by $\mathcal{T}_{22}$. (see eq.(70).)

In eq.(44), $A^{(1)}$ and $A^{(2)}$ are expressed diagrammatically as $(\mathrm{r}-1) \sim(\mathrm{r}-4)$ and (s-1,2) in Fig. 8 , and $A^{(3)}, A^{(4)}$, and $A^{(5)}$ are expressed as $(\mathrm{t}-1 \sim 6)$. The latter contains the momentum derivative of $\gamma(k, \epsilon)$. In eq.(44), the factor 2 due to the spin degeneracy is taken into account.

Finally, we perform the $\epsilon$-integration in eq. (44) under the assumption that $\gamma^{*} \ll T$. In this case, the expression for the MR, eq. (44), becomes much simpler as follows: In the case of $\gamma_{k}^{*} \ll T$, the following replacements are allowed in eq. (44).

$$
\begin{aligned}
& |G|^{4} \rightarrow \pi z_{k} \delta\left(\epsilon+\mu-\epsilon_{k}^{*}\right) \frac{1}{2 \gamma_{k}^{3}}, \\
& |G|^{2} \operatorname{Re}\left\{G^{2}\right\} \rightarrow \pi z_{k} \delta\left(\epsilon+\mu-\epsilon_{k}^{*}\right) \frac{-1}{4 \gamma_{k}^{3}}, \\
& |G|^{4} \operatorname{Im}\left\{G^{R}\right\} \rightarrow \pi z_{k} \delta\left(\epsilon+\mu-\epsilon_{k}^{*}\right) \frac{-3}{8 \gamma_{k}^{4}}, \\
& |G|^{2} \operatorname{Im}\left\{G^{R^{3}}\right\} \rightarrow \pi z_{k} \delta\left(\epsilon+\mu-\epsilon_{k}^{*}\right) \frac{1}{8 \gamma_{k}^{4}}, \\
& |G|^{6} \rightarrow \pi z_{k} \delta\left(\epsilon+\mu-\epsilon_{k}^{*}\right) \frac{3}{8 \gamma_{k}^{5}},
\end{aligned}
$$

By using these relations (45), we see that ( $\mathrm{r}-1)$ and ( $\mathrm{s}-1)$ in Fig. 8 are equal, whereas (r-2) and (s-2) cancel out completely in the case of $\gamma_{k}^{*} \ll T$. After the long but straightforward calculation, we get the following simple result for the MC.

$$
\begin{aligned}
\Delta \sigma_{\mu \mu}^{a} & =B^{2} \cdot \frac{e^{4}}{4 v_{B}} \sum_{\mathbf{k}} \int d \epsilon\left(-\frac{\partial f}{\partial \epsilon}\right) z_{\mathbf{k}} \delta\left(\epsilon+\mu-\epsilon_{\mathbf{k}}^{*}\right) \cdot \frac{J_{\mathbf{k} \mu}}{\gamma_{\mathbf{k}}}\left(\hat{e}_{z} \times \vec{v}_{\mathbf{k}}\right) \cdot \vec{\nabla}\left\{\frac{1}{\gamma_{\mathbf{k}}}\left(\hat{e}_{z} \times \vec{v}_{\mathbf{k}}\right) \cdot \vec{\nabla}\left(\frac{J_{\mathbf{k} \mu}}{\gamma_{\mathbf{k}}}\right)\right\} \\
& =B^{2} \cdot \frac{e^{4}}{4 v_{B}} \oint_{\mathrm{FS}} d S_{k} \frac{J_{\mathbf{k} \mu}}{\gamma_{\mathbf{k}}} \frac{\partial}{\partial k_{\|}}\left\{\frac{\left|\vec{v}_{\mathbf{k}}\right|}{\gamma_{\mathbf{k}}} \frac{\partial}{\partial k_{\|}}\left(\frac{J_{\mathbf{k} \mu}}{\gamma_{\mathbf{k}}}\right)\right\} \\
& =-B^{2} \cdot \frac{e^{4}}{4 v_{B}} \oint_{\mathrm{FS}} d S_{k} \frac{\left|\vec{v}_{\mathbf{k}}\right|}{\gamma_{\mathbf{k}}} \cdot\left(\frac{\partial}{\partial k_{\|}}\left(\frac{J_{\mathbf{k} \mu}}{\gamma_{\mathbf{k}}}\right)\right)^{2},
\end{aligned}
$$

where we used the relation $\left(\hat{e}_{z} \times \vec{v}_{k}\right) \cdot \vec{\nabla}=v_{x} \partial_{y}-v_{y} \partial_{x}=\left|\vec{v}_{k}\right| \frac{\partial}{\partial k_{\|}}$. Here $k_{\|}$is the momentum along $\hat{e}_{\|} \equiv\left(\hat{e}_{z} \times \vec{v}_{k}\right)$, that is, $k_{\|}$is along the Fermi surface on the $x y$-plane. We also used the replacement:

$$
\sum_{k} z_{k} \delta\left(\mu-\epsilon_{k}^{*}\right) \rightarrow \int_{\mathrm{FS}} \frac{1}{\left|\vec{v}_{k}\right|} d S_{k} \quad \text { in 3D. }
$$

On the other hand, in the two-dimensional case,

$$
\sum_{k} z_{k} \delta\left(\mu-\epsilon_{k}^{*}\right) \rightarrow \int_{\mathrm{FS}} \frac{1}{\left|\vec{v}_{k}\right|} d k_{\|} \quad \text { in } 2 \mathrm{D} .
$$

The result (46) is nothing but the result of the relaxation time approximation given by eq.(何), if we replace $2 \tau_{k} \rightarrow \gamma_{k}^{-1}$. In conclusion, the MC derived in this section, $\Delta \sigma_{x x}^{a}$, is of order $\gamma_{k}^{-3}$.

\section{MAGNETOCONDUCTIVITY: WITH FULL VERTEX CORRECTIONS}

In the previous section, we derived the MC by neglecting the vertex corrections as the first step. The obtained expression becomes equal to the result of the relaxation time approximation. However, it will not be applicable for 
(i)

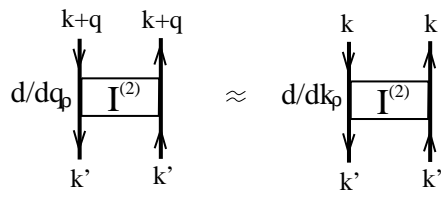

(ii)
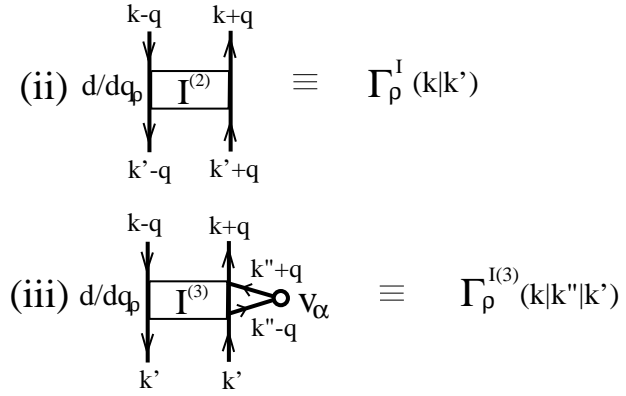

(iv)

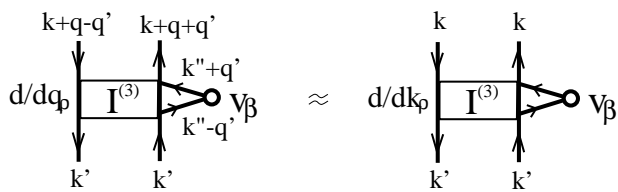

FIG. 11. $q$-derivative of several types of the irreducible vertices contained in $\Phi_{\mu \nu}^{(2)}\left(2 q+2 q^{\prime} ; \omega_{\lambda}\right):(\mathrm{i}) \Gamma^{I}\left(k_{+} ; k_{+} \mid k^{\prime} ; k^{\prime}\right),($ ii) $\Gamma^{I}\left(k_{-} ; k_{+} \mid k_{+}^{\prime} ; k_{-}^{\prime}\right)$, (iii) $\Gamma^{I(3)}\left(k_{-} ; k_{+}\left|k^{\prime} ; k^{\prime}\right| k_{+}^{\prime \prime} ; k_{-}^{\prime \prime}\right) \cdot \partial_{\alpha}^{\prime \prime} G\left(k^{\prime \prime}\right)$, and (iv) $\Gamma^{I(3)}\left(k_{+-} ; k_{++}\left|k^{\prime} ; k^{\prime}\right| k_{0+}^{\prime \prime} ; k_{0-}^{\prime \prime}\right) \cdot \partial_{\beta}^{\prime \prime} G$. Only the $q$-derivative of (i) and (iv) can give the $O\left(\gamma^{-3}\right)$ contributions for the MC. Whereas (ii) and (iii) give at most $O\left(\gamma^{-2}\right)$, which will be explained in Appendix B.

correlated electron systems because the vertex corrections are indispensable to satisfy the conservation laws. In this section, we derive the exact expression for the MC in the Fermi liquid with arbitrary interaction, up to the most divergent contribution with respect to $\gamma^{-1}$.

At first, we take the $q_{\rho} q_{\rho^{\prime}}^{\prime}$-derivative of $\Phi^{(2)}\left(2 q+2 q^{\prime}, \omega_{\lambda}\right)$ which is shown in Figs. 3 and 4 . Now, we should remind that all the diagrams are composed of (a) the bare vertices $v_{\nu}^{0}(k), v_{\mu \alpha}^{0}(k)$, etc, (b) the two point Green functions $G(k, \epsilon)$, and (c) the irreducible four, six and eight vertices $\Gamma^{I}, \Gamma^{I(3)}$ and $\Gamma^{I(4)}$. (see Fig.1.) To obtain the exact result, we have to take the $q_{\rho}, q_{\rho^{\prime}}^{\prime}$-derivative of the every element of $(a)-(c)$ included in $\Phi^{(2)}\left(2 q+2 q^{\prime}, \omega_{\lambda}\right)$ once, by taking care of the momentum suffixes.

The $q_{\rho}\left(q_{\rho^{\prime}}^{\prime}\right)$-derivative of (b) is already shown in Fig. 6. So we consider on the derivative of (c): Figure 11 shows several types of the irreducible vertices which are included in $\Phi^{(2)}\left(2 q+2 q^{\prime}, \omega_{\lambda}\right)$. In the case of (i) and (iv), $\partial / \partial q_{\rho}$ can be replaced by $\partial / \partial k_{\rho}$, while it is impossible for (ii) and (iii). In this section, we consider only the $q_{\rho}$-derivative of (i) and (iv) and neglect that of (ii) and (iii), because they turn out to give less singular contributions with respect to $\gamma^{-1}$ : We will give the explanation for this fact in Appendix B.

From now on, we derive $\left(\partial^{2} / \partial q_{\rho} \partial q_{\rho^{\prime}}^{\prime}\right) \Phi^{(2)}\left(2 q+2 q^{\prime}, \omega_{\lambda}\right)$ by taking care of the definitions of $\Gamma$ and $\Lambda$ given in Fig. 1. This procedure is not easy because we meet hundreds of diagrams in total. For example, in Fig. 13, we show 'all the terms' come from the $q q^{\prime}$-derivative of (a) and (d) in Fig. 3, and (g-1 4), (h-3,4) in Fig. 1 . The definition of the operation $\oplus$ and $\ominus$ in each diagram is given in Fig. 14. As for the $q, q^{\prime}$-derivative of $\Gamma$, only (i) and (iv) in Fig.11 are taken into account. In Fig. 13, we write its sign and the factor above each diagram.

In Fig. 13, (a) does not have the momentum derivative of $\Gamma$ because we drop the terms with (ii) and (iii) in Fig.11. On the other hand, (d) contains $\partial_{\rho^{\prime}}^{\prime} \Lambda_{\mu},(\mathrm{g}-1 \sim 4)$ contains $\partial_{\rho} \Gamma$, and (h-3,4) have the six-point vertex $\Gamma \underline{\alpha}$, respectively. Hereafter, we write $\partial_{\alpha} \Lambda_{\nu}(k) \equiv \Lambda_{\nu, \alpha}(k)$ and $\partial_{\alpha \beta} \Lambda_{\nu}(k) \equiv \Lambda_{\nu, \alpha \beta}(k)$, respectively. Some of the diagrams in Fig. 13 are expressed as

$$
\begin{aligned}
& \text { 1st of (a) }=-T^{2} \sum_{k k^{\prime}, \epsilon \epsilon^{\prime}} \Lambda_{\mu}(k)\left[G \stackrel{\leftrightarrow}{\partial_{\rho}} G^{+}\right] \cdot \Gamma\left(k \mid k^{\prime}\right) \cdot\left[G \stackrel{\leftrightarrow}{\partial_{\rho^{\prime}}^{\prime}} G^{+}\right] \Lambda_{\nu, \alpha \beta}\left(k^{\prime}\right), \\
& 3 r d \text { of }(\mathrm{a})=+T \sum_{k, \epsilon} \Lambda_{\mu}(k)\left[\partial_{\rho^{\prime}} G \cdot \partial_{\rho} G^{+}+\left\langle\rho \leftrightarrow \rho^{\prime}\right\rangle\right] \Lambda_{\nu, \alpha \beta}(k),
\end{aligned}
$$




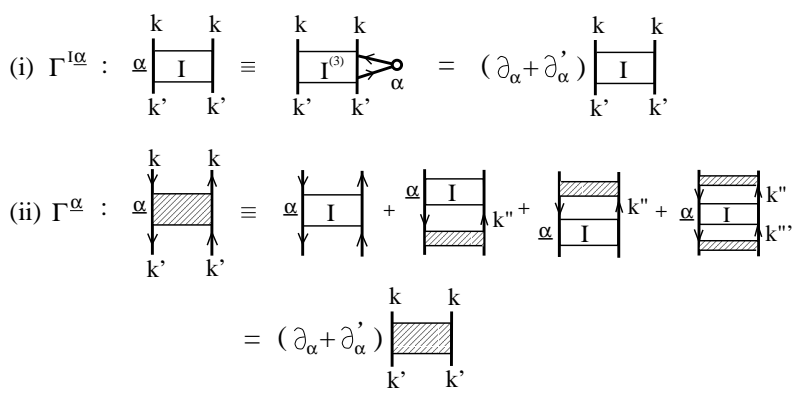

FIG. 12. The definitions of $\Gamma^{I \underline{\alpha}}\left(k \mid k^{\prime}\right)$ and $\Gamma^{\underline{\alpha}}\left(k \mid k^{\prime}\right)$. According to the Ward identity, they are equal to $\left(\partial_{\alpha}+\partial_{\alpha}^{\prime}\right) \Gamma^{I}$ and $\left(\partial_{\alpha}+\partial_{\alpha}^{\prime}\right) \Gamma$, respectively.

$$
\begin{aligned}
\text { 5th of }(\mathrm{a})= & -T \sum_{k, \epsilon} \Lambda_{\mu}(k)\left\{G\left[\partial_{\rho \rho^{\prime}} G^{+}\right]_{(3)}+\left[\partial_{\rho \rho^{\prime}} G\right]_{(3)} G^{+}\right\} \Lambda_{\nu, \alpha \beta}(k), \\
\text { 1st of }(\mathrm{g}-1 \sim 4)= & -T^{2} \sum_{k k^{\prime}, \epsilon \epsilon^{\prime}} \Lambda_{\mu}(k)\left[G \stackrel{\leftrightarrow}{\partial_{\alpha}} G^{+}\right] \cdot \partial_{\rho} \Gamma\left(k \mid k^{\prime}\right) \cdot\left[G \stackrel{\leftrightarrow}{\partial} G^{\prime} G^{+}\right] \Lambda_{\nu, \rho^{\prime}}\left(k^{\prime}\right), \\
\text { 2nd of }(\mathrm{g}-1 \sim 4)= & +T^{3} \sum_{k k^{\prime} k^{\prime \prime}, \epsilon \epsilon^{\prime} \epsilon^{\prime \prime}} \Lambda_{\mu}(k)\left[G \stackrel{\leftrightarrow}{\partial_{\rho}} G^{+}\right] \cdot \Gamma\left(k \mid k^{\prime}\right) \cdot\left[\partial_{\alpha}^{\prime}\left\{G G^{+}\right\}\right] \\
& \times \Gamma\left(k^{\prime} \mid k^{\prime \prime}\right) \cdot\left[G \stackrel{\leftrightarrow}{\partial}{ }_{\beta}^{\prime \prime} G^{+}\right] \Lambda_{\nu, \rho^{\prime}}\left(k^{\prime \prime}\right), \\
\text { 1st of }(\mathrm{h}-3,4)= & +T^{2} \sum_{k k^{\prime}, \epsilon \epsilon^{\prime}} \Lambda_{\mu}(k)\left[G \stackrel{\leftrightarrow}{\partial}_{\rho} G^{+}\right] \cdot \Gamma \underline{\alpha}\left(k \mid k^{\prime}\right) \cdot\left[G \overleftrightarrow{\partial}_{\beta}^{\prime} G^{+}\right] \Lambda_{\nu, \rho^{\prime}}\left(k^{\prime}\right),
\end{aligned}
$$

where $\partial_{x}^{\prime}=\partial / \partial k_{x}^{\prime}$ and $\partial_{x}^{\prime \prime}=\partial / \partial k_{x}^{\prime \prime} \cdot\left[A \stackrel{\leftrightarrow}{\partial}_{\alpha} B\right] \equiv A \cdot \partial_{\alpha} B-B \cdot \partial_{\alpha} A$, and $[\cdots]_{(3)}$ means to pick up only the terms which is proportional to $G^{3}$. Moreover, we wrote $\Gamma\left(k \epsilon^{+} ; k \epsilon \mid k^{\prime} \epsilon^{\prime}, k^{\prime} \epsilon^{\prime+}\right) \equiv \Gamma\left(k \mid k^{\prime}\right)$, and $\Gamma^{\alpha}\left(k \mid k^{\prime}\right)$ in eq. (54) is defined as

$$
\Gamma^{\underline{\alpha}}\left(k \mid k^{\prime}\right) \equiv T^{2} \sum_{p p^{\prime}}\left(1 / T+\Gamma \cdot G G^{+}\right)_{(k ; p)} \cdot \Gamma^{I \underline{\alpha}}\left(p \mid p^{\prime}\right) \cdot\left(1 / T+G G^{+} \cdot \Gamma\right)_{\left(p^{\prime} ; k^{\prime}\right)}
$$

where $\Gamma^{I \underline{\alpha}}$ is introduced as

$$
\Gamma^{I \underline{\alpha}}\left(k \mid k^{\prime}\right)=T \sum_{k^{\prime \prime} \epsilon^{\prime \prime}} \Gamma^{I(3)}\left(k\left|k^{\prime}\right| k^{\prime \prime}\right) \cdot \partial_{\alpha}^{\prime \prime} G\left(k^{\prime \prime}\right)
$$

Both $\Gamma^{\underline{\alpha}}$ and $\Gamma^{I} \underline{\alpha}$ are shown by Feynman diagrams in Fig. 12 (i) and (ii), respectively.

To derive $\left(\partial^{2} / \partial q_{\rho} \partial q_{\rho^{\prime}}^{\prime}\right) \Phi^{(2)}\left(2 q+2 q^{\prime}, \omega_{\lambda}\right)$, we also have to take the $q, q^{\prime}$-derivative for all other diagrams in Figs 3 and 4. After all, we get hundreds of complicated diagrams for the MC, which is not a practical formula for applications. Fortunately, all the diagrams turn out to be collected into small number of simpler diagrams. From now on, we will prove that they are equivalent to Fig. 15 together with Fig. 8 if $J_{\mu(\nu)}$ is replaced with $\Lambda_{\mu(\nu)}$. The expressions for the diagrams in Fig. 15 are given by

$$
\begin{aligned}
& (\mathrm{u}-1)=-2 T^{2} \sum_{k k^{\prime} \epsilon \epsilon^{\prime}} \Lambda_{\mu}(k)\left[G \stackrel{\leftrightarrow}{\partial_{\alpha}} G^{+}\right] \cdot \partial_{\rho} \Gamma\left(k \mid k^{\prime}\right) \cdot\left[G \stackrel{\leftrightarrow}{\partial}{ }_{\beta}^{\prime} G^{+}\right] \Lambda_{\nu, \rho^{\prime}}\left(k^{\prime}\right), \\
& (\mathrm{v}-1)=-2 T^{2} \sum_{k k^{\prime} \epsilon \epsilon^{\prime}} \Lambda_{\mu}(k)\left[G \stackrel{\leftrightarrow}{\partial_{\alpha}} G^{+}\right] \cdot \partial_{\rho} \Gamma\left(k \mid k^{\prime}\right) \cdot\left[\partial_{\beta}^{\prime} G^{+} \cdot \partial_{\rho^{\prime}}^{\prime} G-\partial_{\rho^{\prime}}^{\prime} G^{+} \cdot \partial_{\beta}^{\prime} G\right] \Lambda_{\nu}\left(k^{\prime}\right) .
\end{aligned}
$$

In the same way, we get $(\mathrm{u}-2)=-\left.(\mathrm{u}-1)\right|_{\alpha \leftrightarrow \rho},(\mathrm{u}-3)=-\left.(\mathrm{u}-1)\right|_{\beta \leftrightarrow \rho^{\prime}}$, and $(\mathrm{u}-4)=-\left.(\mathrm{u}-2)\right|_{\beta \leftrightarrow \rho^{\prime}}$.

The expressions for (r-1) and (s-1) in Fig. 8 after the replacement $J_{\mu(\nu)} \rightarrow \Lambda_{\mu(\nu)}$ are given by

$$
\begin{aligned}
& (\mathrm{r}-1)=T \sum_{k \epsilon} \Lambda_{\mu}(k)\left[\partial_{\rho^{\prime}} G \cdot \partial_{\rho} G^{+}\right] \Lambda_{\nu, \alpha \beta}(k), \\
& (\mathrm{s}-1)=-T \sum_{k \epsilon} \Lambda_{\mu}(k)\left\{\left[\partial_{\rho \rho^{\prime}} G^{+}\right]_{(3)} \cdot G+\left\langle G^{+} \leftrightarrow G\right\rangle\right\} \Lambda_{\nu}(k) .
\end{aligned}
$$


(a) :

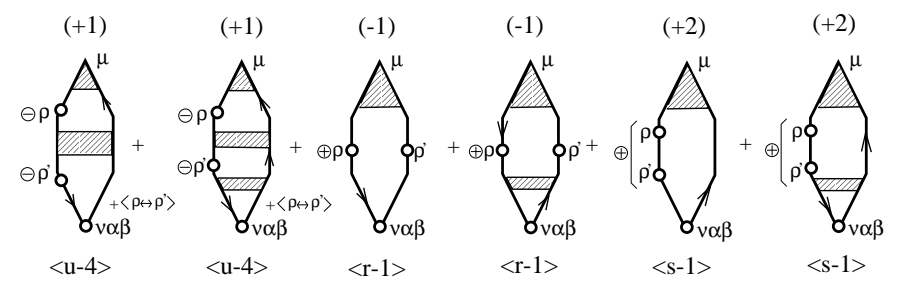

(d)

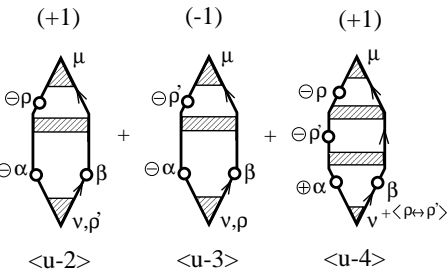

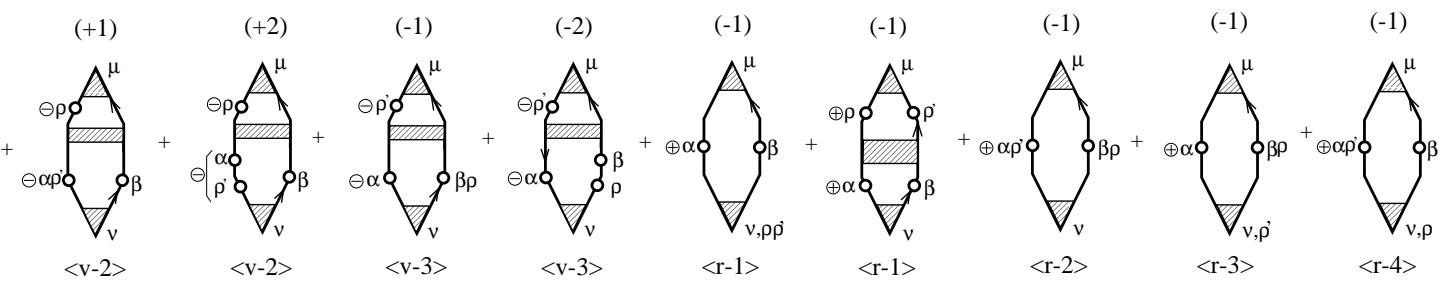
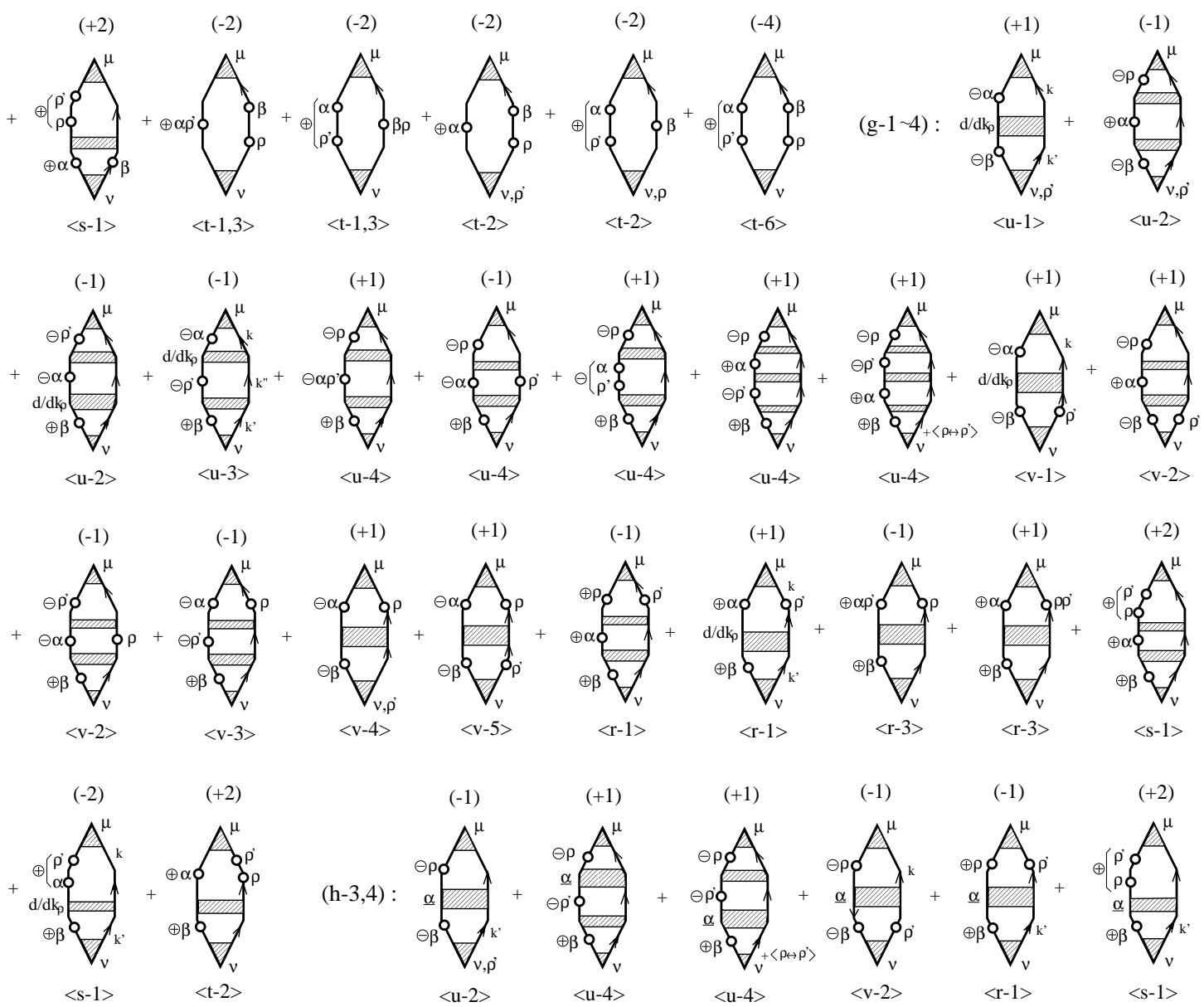

FIG. 13.

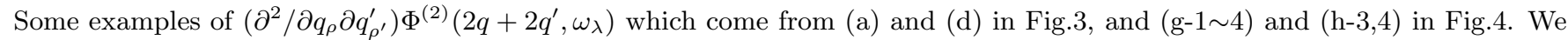
note that the diagrams which are less singular than $O\left(\gamma^{-3}\right)$, e.g., the first term of (i) and (ii) in Fig. 10, are dropped in this figure. Note that the diagrams with the replacement $(\alpha, \rho) \leftrightarrow\left(\beta, \rho^{\prime}\right)$ also exist for $(\mathrm{g}-1 \sim 4)$ and $(\mathrm{h}-3,4)$. Other examples are shown in Appendix C. 


$$
\begin{aligned}
& \oplus \alpha \phi\left|=\alpha+\frac{1}{\phi}\right| \pm \alpha
\end{aligned}
$$

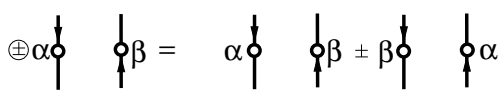

FIG. 14. The definition of the operation $\oplus$ and $\ominus$.

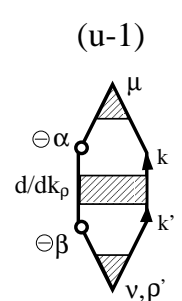

$(+2)$

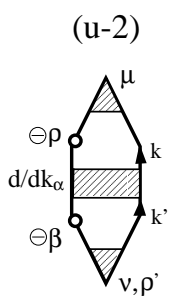

$(-2)$

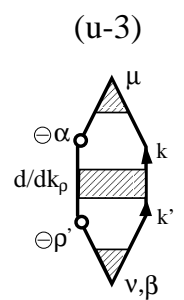

(-2)

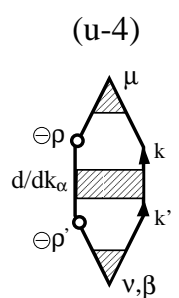

(+2)

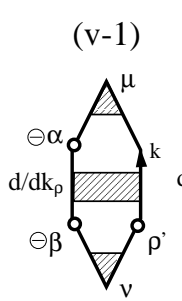

(+2)

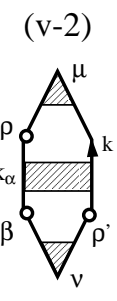

(-2)

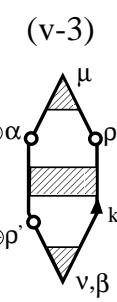

(-2)

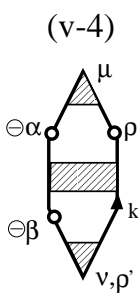

$(+2)$ (v-5)

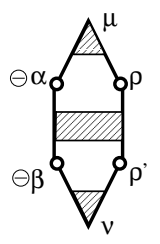

(+2)

FIG. 15. The diagrams for the MC with the $q$-derivative of four-point vertices. The exact MC of order $\gamma^{-3}$ is given by these diagrams together with Fig. 8 . Here, the coefficient \pm 2 comes from the exchange between $(\alpha, \rho) \leftrightarrow\left(\beta, \rho^{\prime}\right)$. 


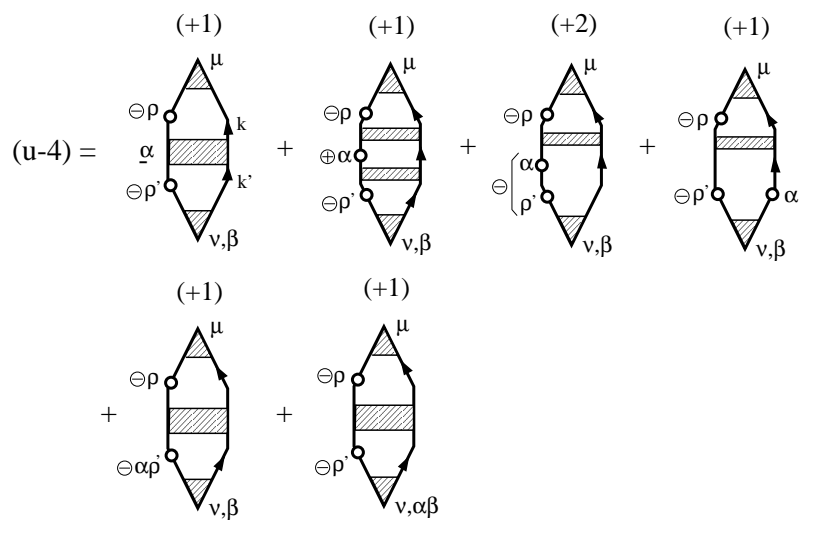

FIG. 16.

The digram for (u-4) in Fig.15 is expanded by using a Ward identity, eq.61).

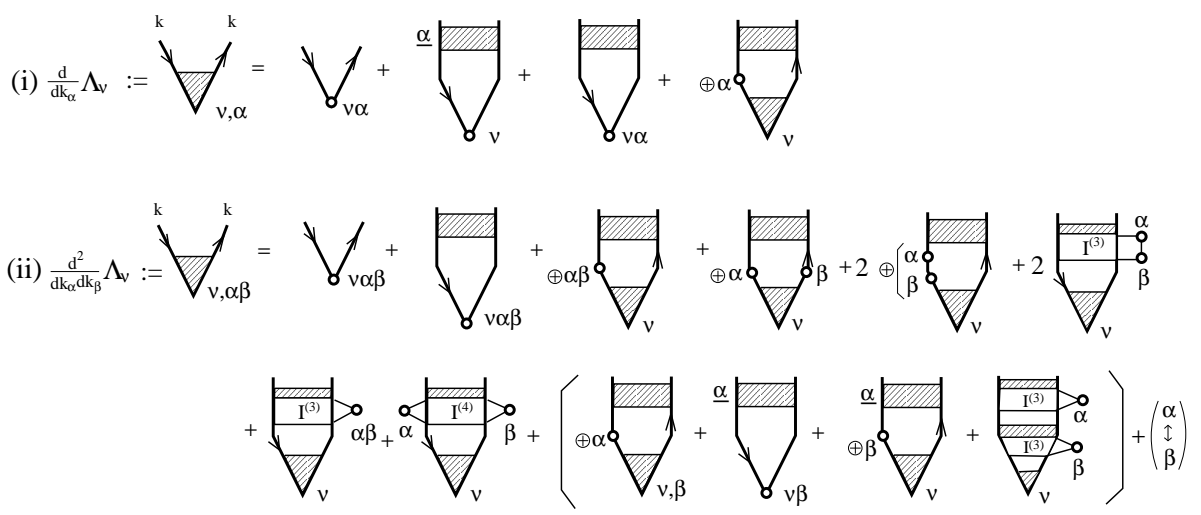

FIG. 17. The diagrammatic expression for (i) $\Lambda_{\nu, \alpha}(k)=\partial_{\alpha} \Lambda_{\nu}(k)$ and (ii) $\Lambda_{\nu, \alpha \beta}(k)=\partial_{\alpha \beta} \Lambda_{\nu}(k)$, respectively.

The procedure of proof is that (i) we decompose the diagrams with $\partial_{\alpha(\beta)}$ or $\partial_{\alpha \beta}$ in Figs. 8 and 1 . by taking several types of Ward identities, and (ii) recognize the one to one correspondence between them and $\left(\partial^{2} / \partial q_{\rho} \partial q_{\rho^{\prime}}^{\prime}\right) \Phi^{(2)}\left(2 q+2 q^{\prime}, \omega_{\lambda}\right)$.

For example, we can show that (u-2) in Fig. 15, is decomposed as Fig. 16 by using a kind of Ward identity as follows:

$$
\begin{aligned}
\partial_{\alpha} \Gamma^{I}\left(k \mid k^{\prime}\right) & =\Gamma^{I \underline{\alpha}}\left(k \mid k^{\prime}\right)-\partial_{\alpha}^{\prime} \Gamma^{I}\left(k \mid k^{\prime}\right), \\
\partial_{\alpha} \Gamma\left(k \mid k^{\prime}\right) & =\Gamma^{\underline{\alpha}}\left(k \mid k^{\prime}\right)+T \sum_{k^{\prime \prime} \epsilon^{\prime \prime}} \Gamma\left(k \mid k^{\prime \prime}\right) \cdot \partial_{\alpha}^{\prime \prime}\{G G\} \cdot \Gamma\left(k^{\prime \prime} \mid k^{\prime}\right)-\partial_{\alpha}^{\prime} \Gamma\left(k \mid k^{\prime}\right),
\end{aligned}
$$

which are shown in Fig.12. To prove these identities, we consider the $k$-derivative of a function $F(k) \equiv$ $\sum_{k^{\prime}} \Gamma\left(k \mid k^{\prime}\right) G\left(k^{\prime}\right)$. Then, $\left(\partial / \partial k_{\alpha}\right) F(k)$ is given by taking the $k$-derivative of every $G$ (i) on the 'connected line' between two $k$-points in $F(k)$ supposing that $k$ runs only on the connected line, and (ii) on all the 'closed loops' after shifting all the momenta by $k$ at the same time virtually. After all, we see that $\left(\partial / \partial k_{\alpha}\right) F(k)$ is given by replacing each one of $G(p)$ 's in $F(k)$ with $\left(\partial / \partial p_{\alpha}\right) G$ and taking the summation of them. Thus, eq.(61) is proved identically. Note that the contribution from (ii) vanishes after all 17]. In the same way, we can derive the Ward identities for $\partial_{\alpha} \Lambda_{\nu}(k ; k) \equiv \Lambda_{\nu, \alpha}$ and $\partial_{\alpha \beta} \Lambda_{\nu}(k ; k) \equiv \Lambda_{\nu, \alpha \beta}$, which are expressed as Fig.17. $\Lambda_{\nu, \alpha \beta}$ contains the irreducible eight-point vertex $\Gamma^{I(4)}$.

We note that $\Lambda_{x, y}(k ; k) \neq \Lambda_{y, x}(k ; k)$ whereas $\Lambda_{\alpha \beta}(k ; k)=\Lambda_{\alpha \beta}(k ; k)$. As for $\Lambda_{\alpha}$, the diagrams with $v_{\alpha}^{0}$ on a closed loop cancel out identically because of the Ward identity, $\Lambda_{\alpha}=\partial_{\alpha}\left(\epsilon_{k}^{0}+\Sigma_{k}\right)$. On the other hand, $\Lambda_{\nu} \neq \partial_{\nu}\left(\epsilon_{k}^{0}+\Sigma_{k}\right)$ because the external frequency $\omega$ is finite in the current study. Because of this fact, the diagrams with $v_{\nu}^{0}$ on a closed loop in $\Lambda_{\nu}$ do not vanish.

By using the identity in Fig. 17, (u-4) in Fig. 16 (with $\partial_{\alpha} \Gamma$ or $\partial_{\alpha \beta} \Gamma$ ) is decomposed into 42 diagrams which are consist of four, six and eight-point vertices (without $\partial_{\alpha} \Gamma$ and $\partial_{\alpha \beta} \Gamma$ ). In the same way, we can decompose all 
other terms in Fig.15. At the final stage of the proof, we verify the one to one correspondence between them and all the terms of $\left(\partial^{2} / \partial q_{\rho} \partial q_{\rho^{\prime}}^{\prime}\right) \Phi^{(2)}\left(2 q+2 q^{\prime}, \omega_{\lambda}\right)$, with signs and factors. Note that each term in Fig. 13 is included in one of the diagrams $(\mathrm{r}-1) \sim(\mathrm{v}-5)$ in Figs. 8 and 15, as denoted under each term. Especially, all the diagrams for $(\mathrm{u}-1),(\mathrm{v}-1),(\mathrm{v}-4)$ and $(\mathrm{v}-5)$ are already appeared in Fig.13. In Appendix C, we present the group of the terms in $\left(\partial^{2} / \partial q_{\rho} \partial q_{\rho^{\prime}}^{\prime}\right) \Phi^{(2)}\left(2 q+2 q^{\prime}, \omega_{\lambda}\right)$ which gives (u-2 4) and (v-2,3) precisely. In the same way, one can check that the rest of terms in $\left(\partial^{2} / \partial q_{\rho} \partial q_{\rho^{\prime}}^{\prime}\right) \Phi^{(2)}\left(2 q+2 q^{\prime}, \omega_{\lambda}\right)$ give $(\mathrm{r}-1 \sim 4),(\mathrm{s}-1,2)$ and $(\mathrm{t}-1 \sim 6)$ precisely.

In conclusion, we have proved that $\left(\partial^{2} / \partial q_{\rho} \partial q_{\rho^{\prime}}^{\prime}\right) \Phi^{(2)}\left(2 q+2 q^{\prime}, \omega_{\lambda}\right)$ is exactly given by Figs. 8 and 15 . In the next section, we perform its analytic continuation with respect to $\omega_{\lambda}$, and derive the exact expression for the MC of order $\tau^{3}$.

\section{ANALYTIC CONTINUATION}

In this section, we perform the analytic continuation of $\left(\partial^{2} / \partial q_{\rho} \partial q_{\rho^{\prime}}^{\prime}\right) \Phi^{(2)}\left(2 q+2 q^{\prime}, \omega_{\lambda}\right)$ obtained in the previous sections, and derive the coefficient of the $\omega$-linear term, i.e., $C_{\mu \nu}^{\alpha \rho, \beta \rho^{\prime}}$ in eq.(16). For this purpose, we have to study the analytic continuation of the following two types of functions:

$$
\begin{aligned}
& L_{\mu \nu}^{a}\left(\omega_{\lambda}\right)=T \sum_{\epsilon_{n}} \Lambda_{\mu}\left(\epsilon_{n} ; \omega_{\lambda}\right) \cdot G\left(\epsilon_{n}^{+}\right) G\left(\epsilon_{n}\right) \cdot \Lambda_{\nu}\left(\epsilon_{n} ; \omega_{\lambda}\right), \\
& L_{\mu \nu}^{b}\left(\omega_{\lambda}\right)=T^{2} \sum_{\epsilon_{n}, \epsilon_{n^{\prime}}} \Lambda_{\mu}\left(\epsilon_{n} ; \omega_{\lambda}\right) \cdot G\left(\epsilon_{n}^{+}\right) G\left(\epsilon_{n}\right) \cdot \Gamma\left(\epsilon_{n}^{+}, \epsilon_{n} \mid \epsilon_{n^{\prime}}, \epsilon_{n^{\prime}}^{+}\right) \cdot G\left(\epsilon_{n^{\prime}}^{+}\right) G\left(\epsilon_{n^{\prime}}\right) \cdot \Lambda_{\nu}\left(\epsilon_{n^{\prime}} ; \omega_{\lambda}\right),
\end{aligned}
$$

where we dropped the momentum suffix for simplicity. Figs. 8 and 15 correspond to $L_{\mu \nu}^{a}\left(\omega_{\lambda}\right)$ and $L_{\mu \nu}^{b}\left(\omega_{\lambda}\right)$, respectively. We promise that several symbols for the momentum derivative of Green functions are implicit in eqs.(62) and (63).

After the analytic continuation with respect to $\omega_{\lambda}(\lambda>0)$, the $\epsilon_{n}$ and $\epsilon_{n^{\prime}}$-summations are replaced with the

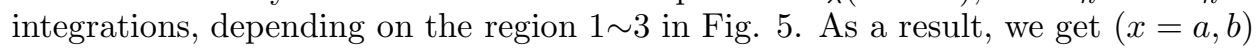

$$
\begin{aligned}
L_{\mu \nu}^{x}(\omega) & =\int \frac{d \epsilon}{4 \mathrm{i} \pi}\left[\operatorname{th} \frac{\epsilon}{2 T} K_{\mu \nu}^{x,(1)}(\epsilon ; \omega)+\left(\operatorname{th} \frac{\epsilon^{+}}{2 T}-\operatorname{th} \frac{\epsilon}{2 T}\right) K_{\mu \nu}^{x,(2)}(\epsilon ; \omega)-\operatorname{th} \frac{\epsilon^{+}}{2 T} K_{\mu \nu}^{x,(3)}(\epsilon ; \omega)\right], \\
K_{\mu \nu}^{a,(i)}(\epsilon ; \omega) & =J_{\mu}^{(i)}(\epsilon ; \omega) g_{i}(\epsilon ; \omega) J_{\mu}^{(i)}(\epsilon ; \omega), \\
K_{\mu \nu}^{b,(i)}(\epsilon ; \omega) & =J_{\mu}^{(i)}(\epsilon ; \omega) g_{i}(\epsilon ; \omega) \cdot T \sum_{k^{\prime}, j=1,2,3} \int \frac{d \epsilon^{\prime}}{4 \pi \mathrm{i}} \mathcal{T}_{i, j}\left(\epsilon^{+} ; \epsilon \mid \epsilon^{\prime} ; \epsilon^{\prime+}\right) g_{j}\left(\epsilon^{\prime} ; \omega\right) J_{\mu}^{(j)}\left(\epsilon^{\prime} ; \omega\right),
\end{aligned}
$$

where we put $i, j=1,2,3$, and $\mathcal{T}_{i, j}\left(\epsilon^{+} ; \epsilon \mid \epsilon^{\prime} ; \epsilon^{\prime+}\right)$ is given by the analytic continuation of $\Gamma\left(\epsilon_{n}^{+} ; \epsilon_{n} \mid \epsilon_{n^{\prime}} ; \epsilon_{n^{\prime}}^{+}\right)$from the region $i$ for $\epsilon_{n}$ and the region $j$ for $\epsilon_{n^{\prime}}$, respectively. Its explicit expression is given in eq.(12) of Ref. [9]. Moreover, $J_{\mu}^{(i)}(k, \epsilon ; \omega)$ is defined as

$$
J_{\mu}^{(i)}(k, \epsilon ; \omega)=v_{\mu}^{0}(k)+\sum_{k^{\prime}, j=1,2,3} \int \frac{d \epsilon^{\prime}}{4 \pi \mathrm{i}} \mathcal{T}_{i j}\left(k \epsilon^{+} ; k \epsilon \mid k^{\prime} \epsilon^{\prime} ; k^{\prime} \epsilon^{\prime+}\right) g_{j}\left(k^{\prime}, \epsilon^{\prime} ; \omega\right) v_{\mu}^{0}\left(k^{\prime}\right) .
$$

Now we put $\omega=0$ in eq.(67) because we study the static conductivity $\sigma(\omega \rightarrow 0)$. In this case, according to the Ward identity [9],

$$
\begin{aligned}
& J_{\mu}^{(1)}(k, \epsilon ; 0)=v_{\mu}(k, \epsilon)-\mathrm{i} \gamma_{\mu}(k, \epsilon), \\
& J_{\mu}^{(3)}(k, \epsilon ; 0)=v_{\mu}(k, \epsilon)+\mathrm{i} \gamma_{\mu}(k, \epsilon) .
\end{aligned}
$$

On the other hand, $J_{\mu}(k, \epsilon) \equiv J_{\mu}^{(2)}(k, \epsilon ; 0)$ can be rewritten as

$$
J_{\mu}(k, \epsilon)=v_{\mu}(k, \epsilon)+\sum_{k^{\prime}} \int \frac{d \epsilon^{\prime}}{4 \pi \mathrm{i}} \mathcal{T}_{22}\left(k \epsilon \mid k^{\prime} \epsilon^{\prime}\right)\left|G\left(k^{\prime}, \epsilon^{\prime}\right)\right|^{2} v_{\mu}\left(k^{\prime}, \epsilon^{\prime}\right),
$$

which is derived by using the following relation satisfied for $|\epsilon| \sim T$ :

$$
v_{\mu}^{0}(k)+\sum_{k^{\prime}, j=1,3} \int \frac{d \epsilon^{\prime}}{4 \pi \mathrm{i}} \mathcal{T}_{2 j}^{(0)}\left(k \epsilon \mid k^{\prime} \epsilon^{\prime}\right) g_{j}\left(k^{\prime}, \epsilon^{\prime} ; 0\right) v_{\mu}^{0}\left(k^{\prime}\right) \approx v_{\mu}(k, \epsilon),
$$


where $\mathcal{T}_{2 j}^{(0)}$ is the 'irreducible' vertex with respect to $g_{2}$-section [9]. Here we denote $\mathcal{T}_{i j}\left(k \epsilon ; k \epsilon \mid k^{\prime} \epsilon^{\prime} ; k^{\prime} \epsilon^{\prime}\right)$ as $\mathcal{T}_{i j}\left(k \epsilon \mid k^{\prime} \epsilon^{\prime}\right)$ for simplicity.

Based on the above argument, we derive the general expression for the MC up to the most singular contribution with respect to $\gamma^{-1}$. At first, we consider the terms in Fig. 8, which correspond to $L_{\mu \nu}^{a}(\omega)$ in eq. (64). By doing the same argument in the previous sections, the term $K_{\mu \nu}^{a,(2)}$ in eq.(64) gives the most singular term, $O\left(\gamma^{-3}\right)$. Thus, the expression for the MC derived from $K_{\mu \nu}^{a,(2)}, \Delta \sigma_{\mu \nu}^{a}$, is given by eq.(44) where $J_{\mu}(k, \epsilon)$ is replaced with eq. (70). This expression is still of order $\gamma^{-3}$ because (the momentum derivative of) $J_{\mu(\nu)}$ is of order $\gamma^{0}$.

Next, we study the terms in Fig. 15, which are expressed as $L_{\mu \nu}^{b}(\omega)$ in eq.(64). In this case, all the terms except $i=j=2$ turn out to vanish identically because eq.(66) contains the following momentum derivatives of $G$ implicitly:

$$
\begin{aligned}
& {\left[G^{R} \stackrel{\leftrightarrow}{\partial}_{\alpha} G^{R}\right]=0,} \\
& \left(\partial_{\alpha} G^{R}\right) \cdot\left(\partial_{\rho} G^{R}\right)-\langle\alpha \leftrightarrow \rho\rangle=0 .
\end{aligned}
$$

In conclusion, we get the expression for $\mathrm{MC}$ which comes from Fig. 15, $\Delta \sigma_{\mu \nu}^{b}$, as follows:

$$
\begin{aligned}
\Delta \sigma_{\mu \nu}^{b}= & B^{2} \cdot \frac{e^{4}}{4 v_{B}} \sum_{k} \int \frac{d \epsilon}{\pi}\left(-\frac{\partial f}{\partial \epsilon}\right) 2\left\{|G|^{2}|\operatorname{Im} G| J_{\mu}\left(v_{x} \partial_{y}-v_{y} \partial_{x}\right)+|G|^{4} W_{\mu}^{(2)}\right\}_{(k, \epsilon)} \\
& \times \sum_{k^{\prime}} \int \frac{d \epsilon^{\prime}}{4 \pi \mathrm{i}} \mathcal{T}_{22}\left(k \epsilon \mid k^{\prime} \epsilon^{\prime}\right) 2\left\{|G|^{2}|\operatorname{Im} G| W_{\nu}^{(1)}+|G|^{4} W_{\nu}^{(2)}\right\}_{\left(k^{\prime}, \epsilon^{\prime}\right)},
\end{aligned}
$$

where

$$
\begin{aligned}
& W_{\mu}^{(1)}(k, \epsilon)=v_{x}(k, \epsilon) J_{\mu, y}(k, \epsilon)-v_{y}(k, \epsilon) J_{\mu, x}(k, \epsilon), \\
& W_{\mu}^{(2)}(k, \epsilon)=\left(-v_{x}(k, \epsilon) \gamma_{y}(k, \epsilon)+v_{y}(k, \epsilon) \gamma_{x}(k, \epsilon)\right) J_{\mu}(k, \epsilon),
\end{aligned}
$$

where $J_{\mu}(k, \epsilon)$ is given by eq.(70), and $J_{\mu, x} \equiv \partial_{x} J_{\mu}$ and $J_{\mu, x y} \equiv \partial_{x y} J_{\mu}$. Moreover, $\partial_{x}\left(\epsilon_{k}^{0}+\Sigma^{R}(k, \epsilon)\right) \equiv v_{x}(k, \epsilon)-$

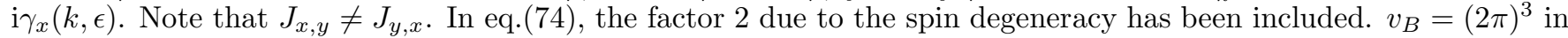
the cubic lattice.

Equation (74) can be rewritten by performing the partial integration with respect to $k$. After the reformation of the equation, we get the following simpler form:

$$
\begin{aligned}
\Delta \sigma_{\mu \nu}^{b}= & -B^{2} \cdot \frac{e^{4}}{4 v_{B}} \sum_{k} \int \frac{d \epsilon}{\pi}\left(-\frac{\partial f}{\partial \epsilon}\right) 2\left\{|G|^{2}|\operatorname{Im} G| W_{\mu}^{(1)}-2|G|^{2} \operatorname{Re} G^{2} W_{\mu}^{(2)}\right\}_{(k, \epsilon)} \\
& \times \sum_{k^{\prime}} \int \frac{d \epsilon^{\prime}}{4 \pi \mathrm{i}} \mathcal{T}_{22}\left(k \epsilon \mid k^{\prime} \epsilon^{\prime}\right) 2\left\{|G|^{2}|\operatorname{Im} G| W_{\nu}^{(1)}+|G|^{4} W_{\nu}^{(2)}\right\}_{\left(k^{\prime}, \epsilon^{\prime}\right)} .
\end{aligned}
$$

The diagrammatic expression is shown in Fig.18. They are of order $\gamma^{-3}$ because the factor $\gamma^{-2}$ comes from each $g_{2}$-lines, and $\mathcal{T}_{22}$ is of order $\gamma$. by

In conclusion, the general expression for the MC in the Fermi liquid system, which is exact in order $\gamma^{-3}$, is given

$$
\Delta \sigma_{x x}=\Delta \sigma_{x x}^{a}+\Delta \sigma_{x x}^{b}
$$

where $\Delta \sigma_{\mu \nu}^{a}$ is given by eq. (44) where $J_{\mu(\nu)}$ is given by eq.(70).

Next, we derived the simplified the expression of eq.(77) under the condition $\gamma^{*} \ll T$. In this case, $\Delta \sigma_{x x}^{a}$ is given by eq. (46) where $J_{\mathbf{k} \mu(\nu)}$ is replaced with $J_{\mu(\nu)}(k, \epsilon=0)$ in eq.(70). As for eq.(76), the following replacement are allowed: $|G|^{2}|\operatorname{Im} G| \rightarrow \pi z_{k} \delta\left(\epsilon+\mu-\epsilon_{k}^{*}\right) / 2 \gamma_{k}^{2},|G|^{2} \operatorname{Re} G^{2} \rightarrow-\pi z_{k} \delta\left(\epsilon+\mu-\epsilon_{k}^{*}\right) / 4 \gamma_{k}^{3}$, and $|G|^{4} \rightarrow \pi z_{k} \delta\left(\epsilon+\mu-\epsilon_{k}^{*}\right) / 2 \gamma_{k}^{3}$. Then, we can perform the energy integrations in eq.(76) easily.

After the long but straightforward calculation, we obtain the following much compact expression for the MC for $\gamma^{*} \ll T:$

$$
\begin{aligned}
& \Delta \sigma_{\mu \nu}=-B^{2} \cdot \frac{e^{4}}{4 v_{B}} \oint_{\mathrm{FS}} \frac{d S_{k}}{\left|\vec{v}_{k}\right|} \frac{1}{\gamma_{k}} d_{\mu}(k) D_{\nu}(k), \\
& d_{\mu}(k)=\left(v_{x}(k, 0) \frac{\partial}{\partial k_{y}}-v_{y}(k, 0) \frac{\partial}{\partial k_{x}}\right) \cdot\left(\frac{J_{\mu}(k, 0)}{\gamma(k, 0)}\right)
\end{aligned}
$$




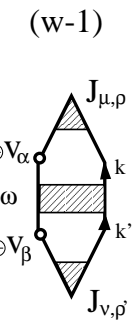

$(-2)$ $(\mathrm{w}-2)$

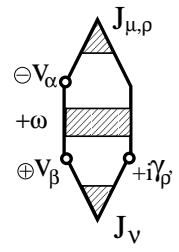

$(-2)$ $(\mathrm{w}-3)$

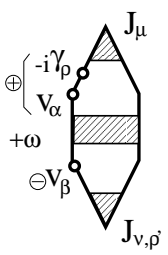

$(-4)$ $(w-4)$

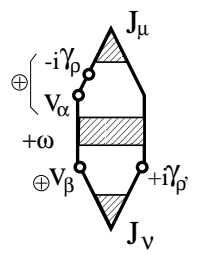

$(-4)$

$+(\alpha \leftrightarrow \rho),\left(\beta \leftrightarrow \rho^{\prime}\right) \quad$ with sign change

FIG. 18.

The expressions for $\Delta \sigma_{\mu \nu}^{b}$ in eq.(76). They are of order $\gamma^{-3}$.

$$
\begin{aligned}
& =W_{\mu}^{(1)}(k, 0) \gamma^{-1}(k, 0)+W_{\mu}^{(2)}(k, 0) \gamma^{-2}(k, 0), \\
D_{\mu}(k) & =\frac{1}{v_{B}} \sum_{k^{\prime}} \int \frac{d \epsilon^{\prime}}{4 \pi \mathrm{i}} \mathcal{T}_{22}\left(k 0 \mid k^{\prime} \epsilon^{\prime}\right)\left|G_{k^{\prime}}\left(\epsilon^{\prime}\right)\right|^{2} d_{\mu}\left(k^{\prime}\right)+d_{\mu}(k) \\
& =\frac{1}{v_{B}} \oint_{\mathrm{FS}} \frac{d S_{k^{\prime}}}{\left|\vec{v}_{k^{\prime}}\right|} \int \frac{d \epsilon^{\prime}}{4 \mathrm{i}} \mathcal{T}_{22}\left(k 0 \mid k^{\prime} \epsilon^{\prime}\right) \frac{1}{\gamma_{k^{\prime}}} d_{\mu}\left(k^{\prime}\right)+d_{\mu}(k),
\end{aligned}
$$

where $\int_{\mathrm{FS}} d S_{k}$ represents the two-dimensional integration on the Fermi surface. Both $D_{\mu}(k)$ and $d_{\mu}(k)$ are real because $\mathcal{T}_{22}$ is pure imaginary for $\omega=0$.

On the other hand, the $\mathrm{MC}$ within the relaxation time approximation is given by

$$
\begin{aligned}
\Delta \sigma_{\mu \nu}^{\mathrm{RTA}} & =-B^{2} \cdot \frac{e^{4}}{4 v_{B}} \oint_{\mathrm{FS}} \frac{d S_{k}}{\left|\vec{v}_{k}\right|} \frac{1}{\gamma_{k}} d_{\mu}^{0}(k) d_{\nu}^{0}(k), \\
d_{\mu}^{0}(k) & =\left(v_{x}(k, 0) \frac{\partial}{\partial k_{y}}-v_{y}(k, 0) \frac{\partial}{\partial k_{x}}\right) \cdot\left(\frac{v_{\mu}(k, 0)}{\gamma(k, 0)}\right) .
\end{aligned}
$$

(see eq.(4).) By comparing the exact formula (78) with eq. (79), we see that $v_{\mu, \nu}$ is replaced with $J_{\mu, \nu}$ due to the vertex correction by $\mathcal{T}_{22}$. Moreover, $d_{\mu}$ is replaced with $D_{\mu}$ due to another vertex $\mathcal{T}_{22}$. Conversely, we get $\Delta \sigma_{x x}^{\mathrm{RTA}}$ if we drop all $\mathcal{T}_{22}$ 's in the exact expression for the MC, eq.(78). Note that $D_{\mu}(k)$ is of order $\gamma^{-1}$, as $d_{\mu}(k)$ and $d_{\mu}^{0}(k)$ are, because $\mathcal{T}_{22}$ is of order $\gamma^{1}$. This fact means that $\Delta \sigma_{x x}$ given by eq.(78) is of order $\gamma^{-3}$.

Before concluding this section, we consider a system with four-fold symmetry around the $z$-axis $\left(\vec{e}_{z} \| \vec{B}\right)$. In this case, we get the expression

$$
\Delta \sigma_{x x}=-B^{2} \cdot \frac{e^{4}}{4 v_{B}} \oint_{\mathrm{FS}} \frac{d S_{k}}{\left|\vec{v}_{k}\right|} \frac{1}{\gamma_{k}} \vec{d}_{\perp}(k) \cdot \vec{D}_{\perp}(k) / 2,
$$

where $\vec{d}_{\perp}=\left(d_{x}, d_{y}\right)$ and $\vec{D}_{\perp}=\left(D_{x}, D_{y}\right)$. Equation (80) means that $\Delta \sigma_{x x}$ is independent of the choice of the $x$-axis on the $x y$-plane.

\section{DISCUSSION AND CONCLUSIONS}

\section{A. The Role of the Vertex Corrections}

In this subsection, we analyze the obtained exact formula for MC, eq.(78), and discuss the role of the vertex corrections (VC's) by $\mathcal{T}_{22}$. As shown in eqs. (2), (3) and (78), $\sigma_{x x}, \Delta \sigma_{x y}$ and $\Delta \sigma_{x x}$ have one, two and three $\mathcal{T}_{22}$ 's, respectively. 
At first, we discuss an isotropic correlated electron system without any Umklapp processes. In this case, Yamada and Yosida proved that $J_{\mu}^{(2)}(k, \epsilon ; \omega)$ given by eq. [67) diverges in the static limit $(\omega=0)$ [10. This fact brings the divergence of the static conductivities $\sigma_{x x}, \Delta \sigma_{x y}$ and $\Delta \sigma_{x x}$ even at finite temperatures, reflecting the momentum conservation law. On the other hand, $J_{\mu}^{(2)}(k, \epsilon ; \omega)$ gives the total current with the 'backflow' in the case of $\gamma_{k}^{*} \ll \omega \ll T$, or in the 'zero-sound regime' [17, 18]. In this case $\vec{J}_{\mathbf{k}}$ is finite. According to our formula (78), $\vec{J}_{\mathbf{k}}=C_{\mathrm{U}} \vec{v}_{\mathbf{k}}, \vec{d}_{\mathbf{k}}=C_{\mathrm{U}} \vec{d}_{\mathbf{k}}^{0}$, and $\vec{D}_{\mathbf{k}}=C_{\mathrm{U}}^{2} \vec{d}_{\mathbf{k}}^{0}$, where $C_{\mathrm{U}}$ is the 'Umklapp coefficient' coming from $\mathcal{T}_{22}[10]$. $C_{\mathrm{U}}$ is finite for finite $\omega$. This fact means that $\Delta \rho(\omega)=0$ up to $B^{2}$ even in the zero-sound regime in an isotropic system.

We also discuss the conductivity of an isotropic free electron system with weak disorders, within the self-consistent Born approximation. Then, it is known that $C_{\mathrm{U}}$ coming from $\mathcal{T}_{22}$ becomes the 'geometric factor'. In this approximation, $\gamma=n_{\text {imp }} \sum_{k^{\prime}}\left|V_{k-k^{\prime}}\right|^{2} \pi \rho_{k^{\prime}}(0)$ where $V_{k}$ is the Fourier transformation of the impurity potential. According to the Ward identity, $\mathcal{T}_{22}$ is given by the ladder-type vertex corrections. By solving the Bethe-Salpeter equation, we get $C_{\mathrm{U}}^{-1}=n_{\mathrm{imp}} \sum_{k^{\prime}}\left|V_{k-k^{\prime}}\right|^{2} \pi \rho_{k^{\prime}}(0)\left(1-\cos \theta_{k-k^{\prime}}\right) / 2 \gamma$. In conclusion, the conductivities are given by $\sigma_{x x}=C_{\mathrm{U}} \cdot\left(n e^{2} / 2 m \gamma\right)$, $\Delta \sigma_{x y}=C_{\mathrm{U}}^{2} \cdot\left(n e^{3} / 4 m^{2} \gamma^{2}\right)$ and $\Delta \sigma_{x x}=C_{\mathrm{U}}^{3} \cdot\left(n e^{4} / 8 m^{3} \gamma^{3}\right)$, respectively. As a result, the relations $R_{\mathrm{H}}=1 / n e$ and $\Delta \rho=0$ up to $B^{2}$, which are equal to the results the RTA, hold in the self-consistent Born approximation if we take the VC's into account correctly, because of the cancellation of $C_{\mathrm{U}}$ 's.

Finally, we discuss the role of the VC's in anisotropic systems, which will be much important. For example, the momentum dependence of $\overrightarrow{J_{\mathbf{k}}}$ in nearly AF Fermi liquids becomes singular singular due to the VC's by $\mathcal{T}_{22}$ 国 7 . This fact explains the seemingly non-Fermi liquid behavior of the Hall coefficient in high- $T_{\mathrm{c}}$ cuprates successfully, without assuming any non-Fermi liquid ground state [19].

It is natural to expect that the VC's by $\mathcal{T}_{22}$ are also important for the MR. In high- $T_{\mathrm{c}}$ cuprates, the Kohler's rule $\Delta \rho / \rho \propto \rho^{-2}$ is strongly violated in a wide range of temperatures [20]. Based on the exact expression derived in this paper, we recently studied this long-standing problem by using the spin-fluctuation theory, with including all the VC's to keep the conserving laws [8]. We found the approximate relation $\Delta \rho / \rho \propto \xi_{\mathrm{AF}}^{4} \cdot \rho^{-2}$ due to the VC's by $\mathcal{T}_{22}$ in the presence of $\mathrm{AF}$ fluctuations $\left(\xi_{\mathrm{AF}}\right.$ being the $\mathrm{AF}$ correlation length). We confirmed that the factor $\xi_{\mathrm{AF}}^{4}$, which comes from the VC's, well explains the violation of the Kohler's rule in high- $T_{\mathrm{c}}$ cuprates.

\section{B. Concluding Remarks}

In this paper, we derived the general expression for the MC based on the Fermi liquid theory from the Kubo formula. We treated the vertex corrections in the exact way by following the Ward identities. The obtained expression is given by eq. (77), which is exact up to $O\left(\gamma^{-3}\right)$. This expression can be simplified as eq. $(78)$ in the case of $\gamma^{*} \ll T$. However, we should use eq.(77) to obtain the reliable result when $\gamma_{k}^{*} \lesssim T$ is realized like in high- $T_{\mathrm{c}}$ cuprates. In all these expressions, we put the factor 2 due to the spin-degeneracy, assuming the paramagnetic state without the magnetic field. Fortunately, these obtained expressions do not contain six and eight-point vertices, although the original diagram for $\Phi^{(2)}$ given by Fig. 1 have them. This fact makes the numerical calculation easier.

It is noteworthy that we can calculate the MC in the conserving way by virtue of the obtained formula (77) or (78): In the purpose, we get at first the proper 'irreducible vertex' through the functional derivative; $\hat{\Gamma}^{I} \equiv \delta \hat{\Sigma} / \delta \hat{G}$. Next, we perform the analytic continuation and get the irreducible vertex $\mathcal{T}_{22}^{\mathrm{I}}$. Finally, we calculate the full vertex correction $\mathcal{T}_{22}$ through the integral equation like eq.(20), and input it in eq.(70) for $J_{\mu}(k, \epsilon)$ and in eq.(78) for $D_{\mu}(k)$. This procedure is classified as the 'conserving approximation' developed by Baym and Kadanoff, which is indispensable to derive reliable transport phenomena [12]. In future, we can apply the present work to the study of interesting magnetotransport phenomena in various kinds of strongly correlated electron systems.

In the obtained expression, the $z$-axis is taken to be the direction of the magnetic field $\vec{B}$. We obtain the 'transverse' $\mathrm{MC}$ if we put $\mu=\nu$ on the $x, y$-plane. The transverse MR is given by eq.(11). In the same way, we get the 'longitudinal' $\mathrm{MC}$ if we put $\mu=\nu=z$. In this case, the longitudinal MR is given by $\Delta \rho / \rho=-\Delta \sigma_{z z} / \sigma_{z z}^{0}$ because of $\Delta \sigma_{z x}=0$. In both cases, $\Delta \sigma \sim O\left(\tau^{3}\right)$ and $\Delta \rho / \rho \sim O\left(\tau^{2}\right)$, respectively. In usual three-dimensional systems, transverse MR and

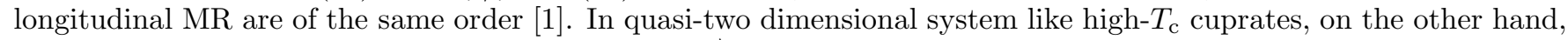
the longitudinal MR on the $a b$-plane in the case of $\vec{B} \| \vec{a},\left(\Delta \rho_{a} / \rho_{a}\right)_{\|}$, is much smaller than the transverse one in the case of $\vec{B} \| \vec{c},\left(\Delta \rho_{a} / \rho_{a}\right)_{\perp}$. Their ratio will be about $O\left(t_{c}^{2} / t_{a}^{2}\right)$, where $t_{c}\left(t_{a}\right)$ is the hopping integral along the $c(a)$-axis.

Because we discussed the MC (MR) up to $O\left(B^{2}\right)$, our formula will be valid under the weak magnetic field, $\max _{k}\left\{\omega_{c} / \gamma_{k}^{*}\right\} \ll 1$, where $\omega_{c} \equiv e B / m c$ is the cyclotron frequency. In general, this condition is satisfied for $B \ll 1$ Tesla in good metals at low temperatures. In optimally-doped high- $T_{\mathrm{c}}$ cuprates, however, $\max _{k}\left\{\omega_{c} / \gamma_{k}^{*}\right\} \ll 1$ will be satisfied even for $B \sim 1$ Tesla because $\max _{k}\left\{1 / \gamma_{k}^{*}\right\} \lesssim T$ is expected above $T_{\mathrm{c}}$. In this article, we did not consider the MR caused by the spin-dependence of $\gamma_{\mathbf{k} \sigma}^{*}$ due to the Zeeman effect, because it is negligible in high- $T_{\mathrm{c}}$ cuprates [20]. However, this spin-effect may cause the negative MR in the vicinity of the ferromagnetic instability [21]. 
Finally, we make short comments on the reliableness of the calculation: We used the MATHEMATICA for the check of some parts of the calculation. Moreover, (1) The gauge invariance of the obtained expression for $\Delta \sigma_{x x}$ is a severe verification of its correctness, because the gauge invariance is violated if we modify only one of the coefficient of diagrams for $\Delta \sigma_{x x}$. We have to mistake at least four coefficients of diagrams accidentally to recover the gauge invariance. (2) The obtained $\Delta \sigma_{x x}$ coincides with $\Delta \sigma_{x x}^{\text {RTA }}$ if we drop all the vertex corrections. (3) The exact expression for $\Delta \sigma_{x y}$ up to $O\left(\tau^{2}\right)$, which was originally obtained in Ref. [3], can be derived much easier by using the technique developed in this article.

\section{acknowledgment}

The author are grateful to D. Vollhardt for enlightening discussions. He is also thankful to K. Yamada, P. Wölfle, W. Metzner and W. Hofstatter for useful comments and discussions. Finally, he thank many participants in 'XII Workshop on Strongly Correlated Electron Systems' at ICTP in Trieste for stimulating discussions.

\section{APPENDIX A: THE HAMILTONIAN AND THE CURRENT IN THE MAGNETIC FIELD}

In this appendix, we derive the expression for eqs. (8), (12) and (13) in $\S$ II. Here we study the one-dimensional system with only the nearest neighbor hopping for simplicity. It is easy to verify that the obtained results A2 - A5) are valid for any dimensional systems with arbitrary range of hoppings.

In the case of $e A \ll 1$, the Peierls phase factor for $t_{l, l+1}$, eq.(可), can be expanded as

$$
\begin{aligned}
\exp \left( \pm \mathrm{i} e\left(A_{l+1}^{\mathrm{tot}}+A_{l}^{\mathrm{tot}}\right) / 2\right)= & 1 \pm \mathrm{i} \frac{e}{2} A\left(e^{\mathrm{i} q}+1\right) e^{\mathrm{i} l q} \pm \mathrm{i} \frac{e}{2} A^{\prime}\left(e^{\mathrm{i} q^{\prime}}+1\right) e^{\mathrm{i} l q^{\prime}} \\
& -\frac{e^{2}}{4} A A^{\prime}\left(e^{\mathrm{i} q}+1\right)\left(e^{\mathrm{i} q^{\prime}}+1\right) e^{\mathrm{i} l\left(q+q^{\prime}\right)} \\
& +\cdots
\end{aligned}
$$

By expanding $t_{i j}$ and $t_{i j}^{*}$ in eq.(6) in the same way, and by performing the Fourier transformation, we get the following result:

$$
\begin{aligned}
H_{B}= & H_{B=0}+e A_{\alpha} \sum_{k} \frac{1}{2}\left(v_{\alpha, k+q / 2}^{0}+v_{\alpha, k-q / 2}^{0}\right) c_{k+q / 2}^{\dagger} c_{k-q / 2}+\left\langle(\alpha, q) \rightarrow\left(\beta, q^{\prime}\right)\right\rangle \\
& +e^{2} A_{\alpha} A_{\beta}^{\prime} \sum_{k}\left(\frac{1}{4} \sum_{s, s^{\prime}}^{-1,1} v_{\alpha \beta, k+\left(s q+s^{\prime} q^{\prime}\right) / 2}^{0}\right) c_{k+\left(q+q^{\prime}\right) / 2}^{\dagger} c_{k-\left(q+q^{\prime}\right) / 2} .
\end{aligned}
$$

By expanding $v_{\alpha, k \pm q / 2}^{0}$ and $v_{\alpha \beta, k \pm\left(q \pm q^{\prime}\right) / 2}^{0}$ in eq. A2) with respect to $q_{\rho}$ and $q_{\rho^{\prime}}^{\prime}$, we see that eq.(\&) in $\S$ II is exact up to $O\left(q, q^{\prime}\right)$ and $O\left(q q^{\prime}\right)$.

Next, we consider the current operator in the magnetic field, whose definition is given by eq.(11). We can derive that

$$
\begin{aligned}
j(m)= & \mathrm{i}\left[H_{B}^{0}, m c_{m}^{\dagger} c_{m}\right] \\
= & \mathrm{i} t \cdot m\left\{e^{\mathrm{i} e\left(A_{m}+A_{m-1}\right) / 2} c_{m-1}^{\dagger} c_{m}-e^{\mathrm{i} e\left(A_{m}+A_{m+1}\right) / 2} c_{m}^{\dagger} c_{m+1}\right. \\
& \left.+e^{-\mathrm{i} e\left(A_{m}+A_{m+1}\right) / 2} c_{m+1}^{\dagger} c_{m}-e^{-\mathrm{i} e\left(A_{m}+A_{m-1}\right) / 2} c_{m}^{\dagger} c_{m-1}\right\}
\end{aligned}
$$

where the integer $m$ denotes the site index. By performing the Fourier transformation, we get

$$
\begin{aligned}
j(p)= & \sum_{m} j(m) e^{\mathrm{i} p m} \\
= & \frac{\partial}{\partial p} \cdot t \sum_{m k k^{\prime}} c_{k}^{\dagger} c_{k^{\prime}} e^{\mathrm{i}\left(-k+k^{\prime}+p\right) m}\left\{e^{\mathrm{i} e\left(A_{m}+A_{m-1}\right) / 2} e^{\mathrm{i} k}-e^{\mathrm{i} e\left(A_{m+1}+A_{m}\right) / 2} e^{\mathrm{i} k^{\prime}}\right. \\
& \left.+e^{-\mathrm{i} e\left(A_{m+1}+A_{m}\right) / 2} e^{-\mathrm{i} k}-e^{-\mathrm{i} e\left(A_{m}+A_{m-1}\right) / 2} e^{-\mathrm{i} k^{\prime}}\right\}
\end{aligned}
$$



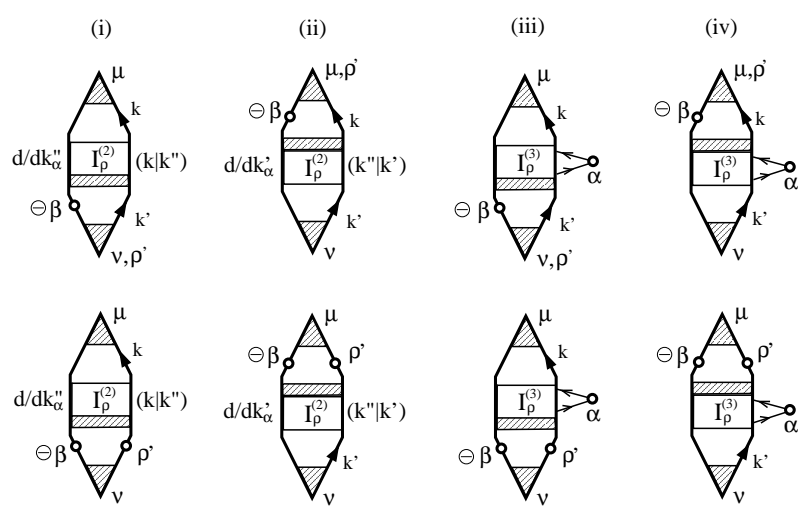

$$
\Gamma_{\rho}^{\mathrm{I}}\left(\mathrm{k} \mid \mathrm{k}^{\prime}\right):=\frac{\mathrm{k}}{\frac{\mathrm{I}_{\rho}^{(2)}}{\mathrm{k}^{\prime}}}\left(\mathrm{k}^{\prime} \mathrm{k}^{\prime}\right) \quad, \quad \Gamma_{\rho}^{\mathrm{I}(3)}\left(\mathrm{k}\left|\mathrm{k}^{\prime \prime}\right| \mathrm{k}^{\prime}\right):=\frac{\mathrm{k}}{\frac{\mathrm{I}_{\rho}^{(3)}}{\mathrm{k}^{\prime}}} \div \mathrm{k}^{\prime \prime}
$$

FIG. 19. The diagrams for the MC of order $\gamma^{-2}$, all of which contain the $q$-derivative of four-point vertices shown in Fig.11 (ii) or (iii). The terms with the exchanges $\beta \leftrightarrow \rho^{\prime}$ with negative signs also exist.

After the straightforward calculation from eq.(A4), we obtain the following expression for $j_{\nu}(p)$ :

$$
\begin{aligned}
j_{\nu}(p)= & \frac{\partial}{\partial p_{\nu}} \sum_{k} c_{k+p / 2}^{\dagger} c_{k-p / 2}\left(\epsilon_{k+p / 2}^{0}-\epsilon_{k-p / 2}^{0}\right) \\
& +\frac{\partial}{\partial p_{\nu}} e A_{\alpha} \sum_{k} c_{k+(p+q) / 2}^{\dagger} c_{k-(p+q) / 2} \cdot \frac{1}{2} \sum_{s}^{-1,1}\left(v_{\alpha \beta, k+(p+s q) / 2}^{0}-v_{\alpha \beta, k-(p+s q) / 2}^{0}\right) \\
& +\left\langle(\alpha, q) \rightarrow\left(\beta, q^{\prime}\right)\right\rangle \\
& +\frac{\partial}{\partial p_{\nu}} e^{2} A_{\alpha} A_{\beta}^{\prime} \sum_{k} c_{k+\left(p+q+q^{\prime}\right) / 2}^{\dagger} c_{k-\left(p+q+q^{\prime}\right) / 2} \cdot \frac{1}{4} \sum_{s, s^{\prime}}^{-1,1}\left(v_{\alpha \beta, k+\left(p+s q+s^{\prime} q^{\prime}\right) / 2}^{0}-v_{\alpha \beta, k-\left(p+s q+s^{\prime} q^{\prime}\right) / 2}^{0}\right) \\
& +\cdots,
\end{aligned}
$$

By expanding $v_{\alpha, k \pm q / 2}^{0}, v_{\alpha \beta, k \pm\left(q \pm q^{\prime}\right) / 2}^{0}$ and $v_{\alpha \beta, k \pm\left(p \pm q \pm q^{\prime}\right) / 2}^{0}$ in eq. $\left.\mathrm{A} 5\right)$ with respect to $q_{\rho}$ and $q_{\rho^{\prime}}^{\prime}$, we can check that eq.(12) in $\S \mathrm{II}$ is exact up to $O\left(q, q^{\prime}\right)$ and $O\left(q q^{\prime}\right)$.

Finally, we comment that another unquestionable definition for the current operator is $\vec{j}=\partial H_{B}^{0} / \partial \vec{A}[13]$. Based on this definition, we can also derive eqs.(12) and (13) exactly up to $O\left(q, q^{\prime}\right)$ and $O\left(q q^{\prime}\right)$.

\section{APPENDIX B: THE MC OF ORDER $\gamma^{-2}$ : THE NEXT SINGULAR CONTRIBUTION}

In this appendix, we study the most divergent terms which come from the $q$-derivative of the irreducible vertices (ii) and (iii) in Fig. 11; $\Gamma_{\rho}^{I}$ and $\Gamma_{\rho}^{I(3)}$. For this purpose, we take the $q q^{\prime}$-derivative of $\Phi^{(2)}\left(2 q+2 q^{\prime}\right)$ given in Figs.3 and 4, and gather all the diagrams which contain $\Gamma_{\rho}^{I}$ or $\Gamma_{\rho}^{I(3)}$, which have been neglected in the discussion in $\S \mathrm{IV}$. This procedure produces a huge number of complicated diagrams. Fortunately, we find that they are collected into eight diagrams shown in Fig. 19, by using the Ward identity. In Fig. 19, only the most divergent terms are presented. This procedure is similar to that developed in $\S I I I$.

For example, the expressions for (i) and (iii) of Fig. 19 are given by,

$$
\begin{aligned}
(\mathrm{i})= & T^{3} \sum_{k k^{\prime} k^{\prime \prime}} \Lambda_{\mu}(k) G G^{+} \cdot \partial_{\alpha}^{\prime \prime} \Gamma_{\rho}^{I}\left(k \mid k^{\prime \prime}\right) \cdot\left(\delta_{k^{\prime \prime}, k^{\prime}} / T+G G^{+} \cdot \Gamma\right) \\
& \cdot\left(\left[G \stackrel{\leftrightarrow}{\partial} G^{\prime} G^{+}\right] \Lambda_{\mu, \rho^{\prime}}\left(k^{\prime}\right)+\left(\partial_{\rho^{\prime}}^{\prime} G \cdot \partial_{\beta}^{\prime} G^{+}-\partial_{\beta}^{\prime} G \cdot \partial_{\rho^{\prime}}^{\prime} G^{+}\right) \Lambda_{\mu}\left(k^{\prime}\right)\right), \\
(\mathrm{iii})= & T^{4} \sum_{k k^{\prime} k^{\prime \prime} k^{\prime \prime \prime}} \Lambda_{\mu}(k) G G^{+} \cdot \Gamma_{\rho}^{I(3)}\left(k\left|k^{\prime \prime}\right| k^{\prime \prime \prime}\right) \cdot \partial_{\alpha}^{\prime \prime \prime} G \cdot\left(\delta_{k^{\prime \prime}, k^{\prime}} / T+G G^{+} \cdot \Gamma\right) \\
& \cdot\left(\left[G \stackrel{\leftrightarrow}{\partial}{ }_{\beta}^{\prime} G^{+}\right] \Lambda_{\mu, \rho^{\prime}}+\left(\partial_{\rho^{\prime}}^{\prime} G \cdot \partial_{\beta}^{\prime} G^{+}-\partial_{\beta}^{\prime} G \cdot \partial_{\rho^{\prime}}^{\prime} G^{+}\right) \Lambda_{\mu}\right),
\end{aligned}
$$




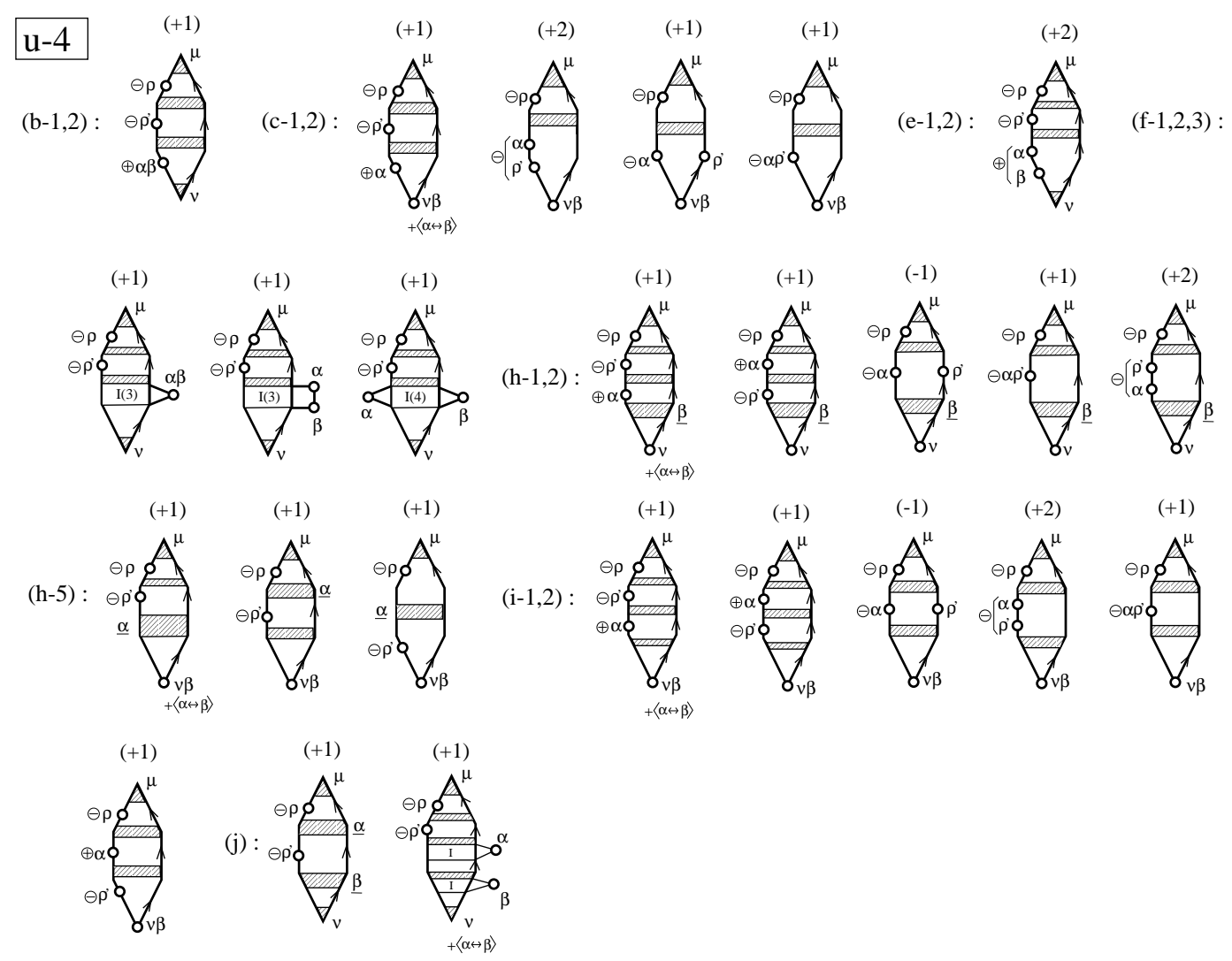

FIG. 20. The part of the diagrams in $\left(\partial^{2} / \partial q_{\rho} \partial q_{\rho^{\prime}}^{\prime}\right) \Phi^{(2)}\left(2 q+2 q^{\prime}, \omega\right)$. These diagrams together with the terms with the notation ' $\langle\mathrm{u}-4\rangle$ ' in Fig. 13 give (u-4) in Fig. 15 .

where $\Gamma_{\rho}^{I}$ and $\Gamma_{\rho}^{I(3)}$ are introduced in Fig. 11. After performing the analytic continuation of them in the same way as $L_{\mu \nu}^{b}$ in eq.(64), the term $G^{+} G \rightarrow G^{R} G^{A}$ gives the most singular contribution. By using the relation (45) together with the fact that $\mathcal{T}_{22}$ is of order $\gamma$, we can clearly show that eqs.(B1) and (B2) are of order $\gamma^{-2}$ In conclusion, we can drop Fig.19 safely in usual metals, where $\gamma_{k}^{*}$ is much smaller than the Fermi energy.

We can also show that Fig.19 gives the exact $O\left(\gamma^{-2}\right)$-terms of the MC, and any diagrams which are not included in Figs.8, 15 and 19 are at most $O\left(\gamma^{-1}\right)$. We note that the expression in Fig.19 should be gauge-invariant because we take all the terms $O\left(\gamma^{-2}\right)$ into account, although it is not written in a gauge-invariant form explicitly.

\section{APPENDIX C: DERIVATION OF FIGS. B AND 15}

In order to derive $\left(\partial^{2} / \partial q_{\rho} \partial q_{\rho^{\prime}}^{\prime}\right) \Phi^{(2)}\left(2 q+2 q^{\prime}, \omega\right)$, we have to take the $q, q^{\prime}$-derivative of all the terms Figs. 3 and 4 . In this appendix, we present the part of them which give $(\mathrm{u}-1 \sim 4)$ and $(\mathrm{v}-1 \sim 5)$ in Fig.15 explicitly. Note that $(\mathrm{u}-1)$, (v-1), (v-4) and (v-5) have already been included in Fig.13.

At first, we concentrate on (u-4) in Fig.15. By using the Ward identity, eq.(61), (u-4) is decomposed into Fig. 16. There, $\Lambda_{\nu, \alpha}$ and $\Lambda_{\nu, \alpha \beta}$ are expressed as Fig. 17, which are also kind of the Ward identities. Thus, (u-4) is decomposed into 42 diagrams in total. On the other hand, Fig. 20 show the part of the terms coming from $\left(\partial^{2} / \partial q_{\rho} \partial q_{\rho^{\prime}}^{\prime}\right) \Phi^{(2)}\left(2 q+2 q^{\prime}, \omega\right)$. Then, we can recognize that the diagrams in Fig. 20 together with the terms with the notation ' $\langle\mathrm{u}-4\rangle$ ' in Fig. 13 give (u-4) precisely, by checking the one-to-one correspondence between them. In the procedure, the symmetry $(\alpha, \rho) \leftrightarrow\left(\beta, \rho^{\prime}\right)$ should be taken into account carefully.

In the next stage, we study (u-2), (u-3), (v-2) and (v-3). As for (u-2) and (v-2), they are decomposed into Fig.21 by using the Ward identity, Eq.(61). Moreover, $\Lambda_{\mu, \alpha(\beta)}(k)=\partial_{\alpha(\beta)} \Lambda_{\mu}(k)$ is expressed diagrammatically in Fig.17. Thus, each (u-2) and (v-2) is decomposed into 9 terms. In the same way, each $(\mathrm{u}-3)$ and $(\mathrm{v}-3)$ is given by 4 terms. On the other hand, Fig. 22 show the part of the terms coming from $\left(\partial^{2} / \partial q_{\rho} \partial q_{\rho^{\prime}}^{\prime}\right) \Phi^{(2)}\left(2 q+2 q^{\prime}, \omega\right)$. Then, we can also see that 


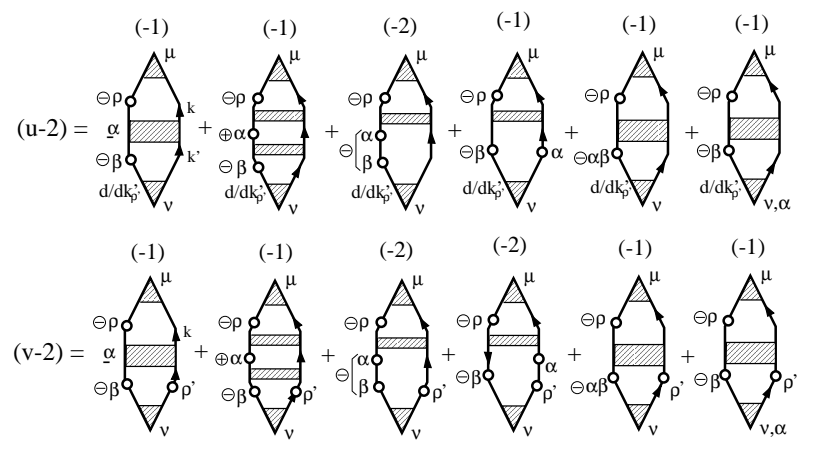

FIG. 21. The expanded expressions for $(\mathrm{u}-2)$ and (v-2) given in Fig. 15. They are derived by using a Ward identity, eq.(61).
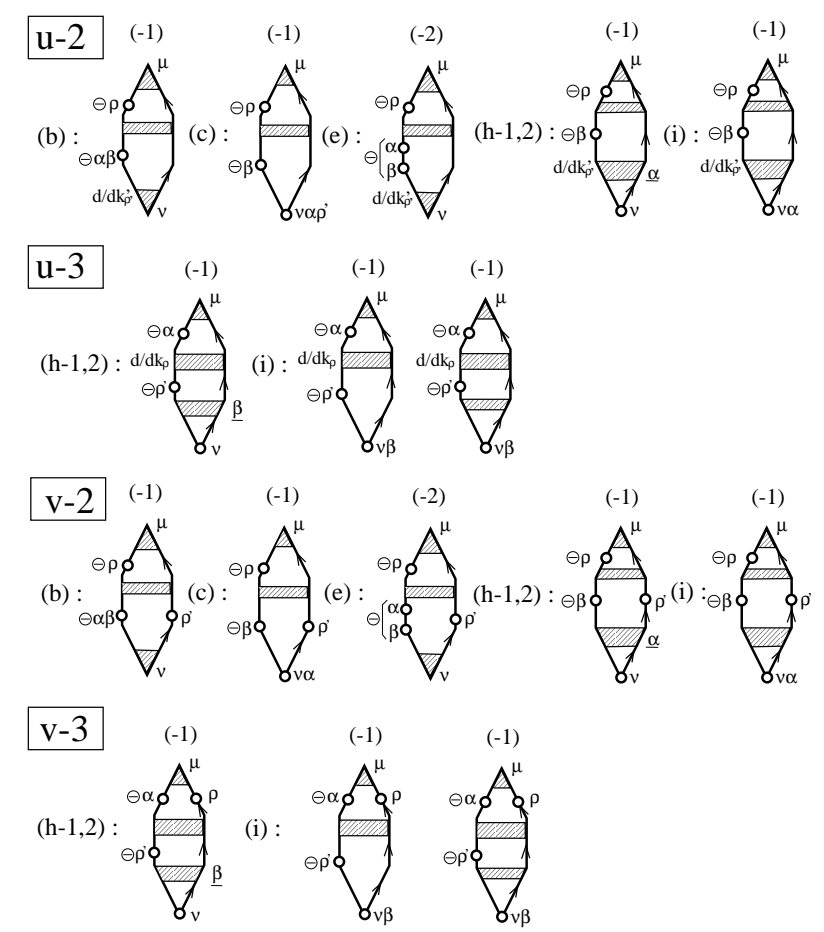

FIG. 22. The part of the diagrams in $\left(\partial^{2} / \partial q_{\rho} \partial q^{\prime}{ }^{\prime}\right) \Phi^{(2)}\left(2 q+2 q^{\prime}, \omega\right)$. These diagrams together with the corresponding terms in Fig 13 give (u-2), (u-3), (v-2) and (v-3) in Fig.15. 


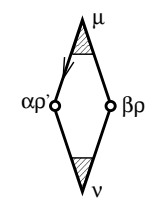

$(-1)$

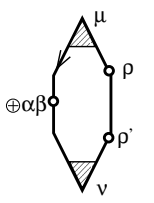

$(+2)$

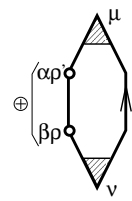

$(-1)$

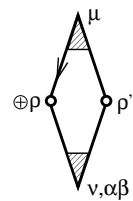

$(-1)$

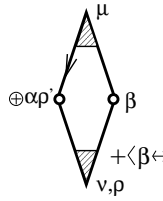

$(-1)$

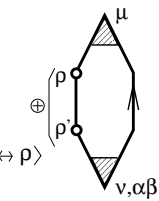

$(+2)$

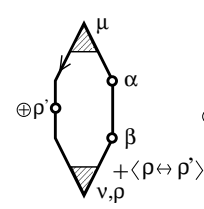

$(+2)$

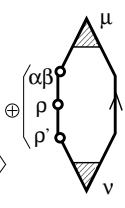

$(+2)$

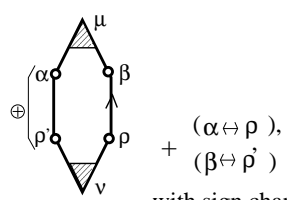

$(-4)$

with sign change

FIG. 23. The rest of the diagrams which come from $\left(\partial^{2} / \partial q_{\rho} \partial q_{\rho^{\prime}}^{\prime}\right) \Phi^{(2)}\left(2 q+2 q^{\prime}, \omega_{\lambda}\right)$. After the analytic continuation of these terms, we get $(\mathrm{r}-1 \sim 4)(\mathrm{s}-1,2)$ and $(\mathrm{t}-1 \sim 6)$ in Fig. 8 .

Fig.22, together with the diagrams with the notations ' $\left.\langle\mathrm{u}-2\rangle\rangle^{\prime}, '\langle\mathrm{u}-3\rangle\right\rangle^{\prime},{ }^{\prime}\langle\mathrm{v}-2\rangle$ ' and ' $\langle\mathrm{v}-3\rangle$ ' in Fig.13, give (u-2), (u-3), (v-2) and (v-3) in Fig.15 precisely. Then, we have to take the symmetry $(\alpha, \rho) \leftrightarrow\left(\beta, \rho^{\prime}\right)$ into account carefully.

In the same way, we can write down all the rest of the terms in $\left(\partial^{2} / \partial q_{\rho} \partial q_{\rho^{\prime}}^{\prime}\right) \Phi^{(2)}\left(2 q+2 q^{\prime}\right.$, $\left.\omega\right)$ which are of order $\gamma^{-3}$. It is rather straightforward to see that they are collected into the 9 terms in Fig.23 precisely. We will not show the detail to avoid redundancy. Next, we perform the analytic continuation of Fig.23 to obtain the expression for the MC. By dropping several terms which turn out to be zero, we get the results $(\mathrm{r}-1 \sim 4)(\mathrm{s}-1,2)$ and $(\mathrm{t}-1 \sim 6)$ in Fig.8 which is of order $\gamma^{-3}$.

In conclusion, $C_{\mu \nu}^{\alpha \rho ; \beta \rho^{\prime}}=\left(\partial^{2} / \partial q_{\rho} \partial q_{\rho^{\prime}}^{\prime}\right) \Phi^{(2)}\left(2 q+2 q^{\prime}, \omega+\mathrm{i} 0\right) / 8$ is exactly given by Figs.8 8 and 15 as for the most singular terms with respect to $\gamma^{-1}$. Because the $\mathrm{MC}$ is given by $\Delta \sigma_{x x}=C_{x x}^{x y ; x y}$, the derived expression for the MC in the present work, eq.(77) or eq.(78), is exact up to $O\left(\gamma^{-3}\right)$.

\section{APPENDIX D: COMMENT ON COND-MAT/0006028}

In recent works, we find the following relations based on the conserving approximation, $\Delta \sigma_{x y} \propto \xi_{\mathrm{AF}}^{2} \gamma^{-2}$ [] and $\Delta \sigma_{x x} \propto \xi_{\mathrm{AF}}^{4} \gamma^{-3}[8]$, where $\xi_{\mathrm{AF}}$ is the AF correlation length, and $\gamma=\operatorname{Im} \Sigma_{k}(-\mathrm{i} \delta)$. They are the most divergent terms with respect to $\gamma^{-1}$. However, in a recent e-preprint [O. Narikiyo, cond-mat/0006028], the author strongly claimed that the above results are inadequate for explaining the non-Fermi liquid behaviors in high- $T_{\mathrm{c}}$ cuprates. His claims will be summarized as follows: (i) The conductivity at finite temperatures given by the conserving approximation remains finite even if no Umklapp processes exist, which is a serious unphysical result. (ii) In the conserving approximation, the Maki-Thompson process violates the Fermi liquid behavior $\rho \propto T^{2}$. (iii) The contribution for $\Delta \sigma_{x y}$ from the momentum derivative of $\gamma_{k}$ is overlooked in Ref. [4] although it is necessary.

In this appendix, we show that the statements (i)-(iii) are inappropriate, so the conserving approximation gives reliable results for high- $T_{\mathrm{c}}$ cuprates as follows.

First, we show that the statement (i), which is the main claim of the author, is just false. Actually, we can show that the conserving approximation based on the FLEX method produces infinite conductivity in the spherical model without Umklapp process: We can prove it straightforwadly by following the Yamada and Yosida's transport theory given by Ref. [10], by taking account of both the Maki-Thompson (MT) and the Aslamazov-Larkin (AL) processes. On the other hand, only the MT-process becomes crucial if the AF fluctuations are strong like in high- $T_{\mathrm{c}}$ cuprates, as shown in Ref. [4.) In this case, the Bethe-Salpeter eq. (6.14) of Ref. [10] is also satisfied by replacing eq.(6.14) with

$$
\Delta_{0}\left(k, k^{\prime} ; k^{\prime}+q, k-q\right)=\pi \rho_{k-q}(0) \rho_{k^{\prime}+q}(0) \rho_{k^{\prime}}(0)\left[(\pi T)^{2}+\epsilon^{2}\right] W_{q}(0),
$$

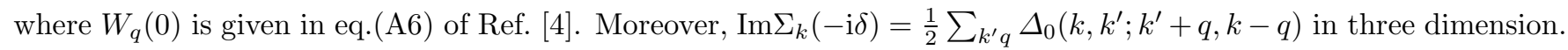
As a result, by following the discussion in Ref. [10], we see that the FLEX approximation can produce the fact that $\sigma_{x x}=\infty$ even for $T>0$ unless the Umklapp processes exist, as a consequence of the momentum conservation law.

It is a commonsense that the conserving laws are quite important for the study of transport phenomena; see Ref. 12]. We found that it is also the case in high- $T_{\mathrm{c}}$ cuprates.

The statement (ii) is inappropriate: The MT process does not prevent the low temperature Fermi liquid behavior $\rho \propto T^{2}$ because the MT process does not give a singular temperature dependence of $\vec{J}_{k}$ at the cold spot. In fact, 
according to the analysis in Ref. 仼, $\vec{J}_{k} \gtrsim \vec{v}_{k} / 2$ at the cold spot due to the MT process is the presence of the strong AF fluctuations. Secondly, $\operatorname{Im} \Sigma_{k}(-\mathrm{i} \delta) \propto T^{2}$ in 3D is ensured in the FLEX approximation at sufficiently low temperatures unless its ground state has a AF order, because the FLEX is classified as the conserving approximation. As a result, the MT process in the conserving approximation does not violate the relation $\rho \propto T^{2}$ in the Fermi liquid regime. (In the numerical results of Ref. [4], the lowest temperature is too high to see the behavior $\rho \propto T^{2}$.)

On the other hand, the Hall coefficient is proportional to the coefficient $\left(\partial \theta_{J} / \partial k_{\|}\right)$at the cold spot. In Refs. 画, 5], we find that the MT process produces a seemingly non-Fermi liquid behavior of $R_{\mathrm{H}}$ at higher temperatures where $\xi_{\mathrm{AF}}$ is strongly temperature dependent. On the other hand, $R_{\mathrm{H}}$ should be nearly constant in the Fermi liquid regime where $\xi_{\mathrm{AF}}$ becomes nearly constant.

The statement (iii) is false; actually, $\Delta \sigma_{x y}$ within the relaxation time approximation is given by

$$
\Delta \sigma_{x y} \propto \oint_{\mathrm{FS}} d k_{\|}\left(\vec{l}_{k} \times \frac{\partial}{\partial k_{\|}} \vec{l}_{k}\right),
$$

where $\vec{l}_{k}=\vec{v}_{k} \gamma_{k}^{-1}$. If we take the vertex corrections into account, $\vec{v}_{k}$ is replaced with $\vec{J}_{k}$ in eq. (D2). Thus, $\Delta \sigma_{x y}$ seems to contain the momentum derivative of $\gamma_{k}$. However, eq.(D2) $\left(\vec{l}_{k}=\vec{J}_{k} \gamma_{k}^{-1}\right)$ is rewritten as

$$
\Delta \sigma_{x y} \propto \oint_{\mathrm{FS}} d k_{\|}\left|\vec{l}_{k}\right|^{2}\left(\frac{\partial \theta_{J}(k)}{\partial k_{\|}}\right)
$$

where $\theta_{J}(k)=\tan ^{-1}\left(J_{x} / J_{y}\right)_{k}=\tan ^{-1}\left(l_{x} / l_{y}\right)_{k}$. (see Ref. 四.) Thus, the momentum derivative of $\gamma_{k}$ does not apprear in eq. (D3). As a result, the calculations of $R_{\mathrm{H}}$ in Refs. [4, 5 ] based on eq. (D3) are correct.

In conclusion, our results of the Hall effect and the magnetorestance based on the conserving approximation are reliable and consistent with experiments. We find that only the MT-type process plays an important role in the transport phenomema in nearly AF Fermi liquid. We note that some vertex corrections are dropped in eq.(3) in Ref.[26] of cond-mat/0006028, which is for quasiprticle contribution to $\Delta \sigma_{x x}$. The correct expression for the MC of order $O\left(\gamma_{k}^{-3}\right)$ is given by eq.(77) in this article, or by eq.(78) in the case of $\gamma_{k}^{*} \ll T$.

[1] J.M. Ziman: Electrons and Phonons (Clarendon Press, ltd., Oxford, 1960).

[2] e.g., B.P. Stojković, D. Pines: Phys. Rev. B 55 (1996) 857.

[3] H. Kohno and K. Yamada: Prog. Theor. Phys. 80 (1988) 623.

[4] H. Kontani, K. Kanki and K. Ueda: Phys. Rev. B 59 (1999) 14723.

[5] K. Kanki and H. Kontani: J. Phys. Soc. Jpn. 68 (1999) 1614.

[6] H. Kontani, K. Kanki and K. Ueda: J. Phys. Chem. Solids 62 (2001) 83.

[7] H. Kontani and H. Kino: Phys. Rev. B 63 (2001) 134524.

[8] H. Kontani: cond-mat/0011327.

[9] G. M. Eliashberg: Sov. Phys. JETP 14 (1962), 886.

[10] K. Yamada and K. Yosida: Prog. Theor. Phys. 76 (1986) 621.

[11] J. Takeda, T. Nishikawa, and M. Sato: Physica C 231 (1994) 293.

[12] G. Baym and L.P. Kadanoff: Phys. Rev. 124 (1961), 287; G. Baym: Phys. Rev. 127 (1962), 1391.

[13] e.g., E. Fradkin: Field Theories of Condenced Matter Physics (Addison-Wesley, Reading 1991), Chap 9.

[14] H. Fukuyama, H. Ebisawa and Y. Wada: Prog. Theor. Phys. 42 (1969) 494.

[15] G.D. Mahan, Many-Particle Physics, 2nd ed. (Plenum Press, New York, 1990), Chap. 1.

[16] R. Kubo: J. Phys. Soc. Jap. 12 (1957) 570.

[17] P. Noziéres: Theory of Interaction Fermion Systems (Benjamin, New York, 1964).

[18] A.A. Abricosov, L.P. Gor'kov and I.E. Dzyaloshinskii: Methods of Quantum Field Theory in Statistical Physics (Dover, New York, 1975).

[19] P.W. Anderson: Phys. Rev. Lett. 67 (1991) 2092.

[20] e.g., J.M. Harris, Y.F. Yan, P. Mati, N.P. Ong, P.W. Anderson, T. Kimura and K. Kitazawa: Phys. Rev. Lett. 75 (1995) 1391.

[21] R. Arita, K. Kuroki, and H. Aoki: cond-mat/991115. 